\title{
Supplement to: Convergence rates of empirical block length selectors for block bootstrap
}

\author{
DANIEL J. NORDMAN and SOUMENDRA N. LAHIRI \\ Department of Statistics \\ Iowa State University \\ Ames, IA 50011 \\ E-mail: dnordman@iastate.edu \\ Department of Statistics \\ Texas A\&M University \\ College Station, TX 77843 \\ E-mail: snlahiri@stat.tamu.edu
}

This supplement provides proofs of the main results (Theorems 2-3) about the convergence rates for the Hall-Horowitz-Jing (HHJ) and non-parametric plug-in (NPPI) methods for block selection from Sections 3-4 of the main manuscript, considering optimal block estimation for the moving block bootstrap (MBB) variance estimator with a smooth function model statistic. Section 7 of the main manuscript describes two auxiliary results (Theorems 5-6) needed to establish the main convergence rates of interest; these auxiliary results are re-iterated and proven in the following.

The supplement consists of Appendices A-B. Appendix A outlines notation (in Section A.1), provides some preliminary results with proofs including Theorem 5 (in Section A.2), and establishes the auxiliary Theorem 6 (in Section A.3). These are required for the proofs of main convergence results, given in Appendix B.

\section{Appendix A: Preliminary results and auxiliary theorems}

Proofs of the main results in Appendix B require a few preliminary results and auxiliary theorems described in Lemmas 1-3 and Theorems 5-6 below (see also Section 7 of the main manuscript).

\section{A.1. Notation}

For $\mathbb{Z}_{+}=\{0,1,2, \ldots\}, \nu=\left(\nu_{1}, \ldots, \nu_{d}\right)^{\prime} \in \mathbb{Z}_{+}^{d}$ and $x=\left(x_{1}, \ldots, x_{d}\right)^{\prime} \in \mathbb{R}^{d}$, let $D^{\nu}$ denote the $\nu$ th order partial differential operator $\partial^{\nu_{1}+\ldots+\nu_{d}} / \partial x_{1}^{\nu_{1}} \ldots \partial x_{d}^{\nu_{d}}$ and write $\|v\|_{1}=$ 
$\sum_{i=1}^{d} \nu_{i}, x^{\nu}=\prod_{i=1}^{d} x_{i}^{\nu_{i}}, \nu !=\prod_{i=1}^{d}\left(\nu_{i} !\right), c_{\nu}=D^{\nu} H(\mu) / \nu !$ and let $\nabla=\left(\partial H(\mu) / \partial x_{1}, \ldots, \partial H(\mu) / \partial x_{d}\right)^{\prime}$ be the vector of first order partial derivatives of $H$ at $\mathrm{E} X_{t}=\mu \in \mathbb{R}^{d}$.

As several results to follow concern the subsampling-based HHJ method, we require notation to express the MBB variance estimator $\hat{\sigma}_{m}^{2}(b)$ based on length $b \in \mathcal{J}_{m}$ blocks from size $m$ samples $\mathcal{X}_{m}=\left(X_{1}, \ldots, X_{m}\right)$. Let $\bar{X}_{i, b}=\sum_{j=i}^{i+b-1} X_{j} / b$ denote the sample average of the $i$ th block, $i=1, \ldots, m-b+1$. Define the MBB sample average $\bar{X}_{m}^{*}=$ $\sum_{j=1}^{m_{1}} \bar{X}_{j, b}^{*} / m_{1}$ where $m_{1}=\lfloor m / b\rfloor, \bar{X}_{j, m}^{*}=\bar{X}_{I_{j}, m}$ and $I_{1}, \ldots, I_{m_{1}}$ are iid discrete uniform variables on $\{1, \ldots, m-b+1\}$. Using Taylor's expansion of $\hat{\theta}_{m}^{*}=H\left(\bar{X}_{m}^{*}\right)$ around $\mathrm{E}_{*} \bar{X}_{m}^{*} \equiv$ $\hat{\mu}_{m}=\sum_{i=1}^{m-b+1} \bar{X}_{i, b} /(m-b+1)$, we may write

$$
\begin{aligned}
& \hat{\sigma}_{m}^{2}(b) \\
= & m_{1} b \operatorname{Var}_{*}\left(L_{m}^{*}\right)+m_{1} b \operatorname{Var}_{*}\left(Q_{m}^{*}\right)+m_{1} b \operatorname{Var}_{*}\left(C_{m}^{*}\right) \\
& +2 m_{1} b \operatorname{Cov}_{*}\left(L_{m}^{*}, Q_{m}^{*}\right)+2 m_{1} b \operatorname{Cov}_{*}\left(L_{m}^{*}, C_{m}^{*}\right)+2 m_{1} b \operatorname{Cov}_{*}\left(Q_{m}^{*}, C_{m}^{*}\right),
\end{aligned}
$$

where $L_{m}^{*}=\hat{\nabla}^{\prime}\left(\bar{X}_{m}^{*}-\hat{\mu}_{m}\right)$ for $\hat{\nabla}=\left(\partial H\left(\hat{\mu}_{m}\right) / \partial x_{1}, \ldots, \partial H\left(\hat{\mu}_{m}\right) / \partial x_{d}\right)^{\prime}, Q_{m}^{*}=\sum_{\|\nu\|_{1}=2} \hat{c}_{\nu}\left(\bar{X}_{m}^{*}-\right.$ $\left.\hat{\mu}_{m}\right)^{\nu}$ defining $\hat{c}_{\nu}=D^{\nu} H\left(\hat{\mu}_{m}\right) / \nu$ !, and $C_{m}^{*}=3 \sum_{\|\nu\|_{1}=3}(\nu !)^{-1}\left(\bar{X}_{m}^{*}-\hat{\mu}_{m}\right)^{\nu} \int_{0}^{1}(1-\omega) D^{\nu} H\left(\hat{\mu}_{m}+\right.$ $\left.\omega\left(\bar{X}_{m}^{*}-\hat{\mu}_{m}\right)\right) d \omega$; here we suppress the dependence of $L_{m}^{*}, Q_{m}^{*}$ and $C_{m}^{*}$ on the block length $b \in \mathcal{J}_{m}$. Due to centering by $\hat{\mu}_{m}$ above, we may (and do) assume throughout that $\mathrm{E} X_{t}=\mu=0$ without loss of generality.

In the following, let $C$ denote a generic constant, which does not depend on any sample sizes $(m, n)$, block sizes $(\ell, b)$ or integer indices. At times, for clarity and brevity, we shall also adopt the notation " $F_{m}(b)=O_{u}\left(m^{a}\right)$ " to denote $\max _{b \in \mathcal{J}_{m}}\left|F_{m}(b)\right|=O\left(m^{a}\right)$ as $m \rightarrow \infty$ for a real-valued function $F_{m}(b), b \in \mathcal{J}_{m}$ and $a \in \mathbb{R}$; that is, order $O_{u}(\cdot)$ denotes "uniform in $b \in \mathcal{J}_{m}$."

\section{A.2. Preliminary results}

Lemma 1 summarizes basic moment bounds and inequalities for later use; parts (i)-(ii) are from Doukhan $\left(1994\right.$, p. 9, 26) ${ }^{1}$ while part (iii) follows from Billingsley (1968, cf. (12.4), (12.9) and Theorem 12.1 there $)^{2}$.

Lemma 1. ( $i)$ For a strictly stationary process $\left\{X_{t}\right\}$ with mean $\mathrm{E} X_{t}=\mu \in \mathbb{R}^{p}$ satisfying condition $M_{r}, \mathrm{E}\left\|\sum_{i=1}^{m}\left(X_{i}-\mu\right)\right\|^{2 \tau} \leq C m^{\tau}$ holds for a constant $C>0$ not depending on $m \geq 1$ or $0 \leq \tau \leq r$.

(ii) If a real-valued random variable $Y_{i}$ is defined with respect to $\left\{X_{t}: t \in A_{i}\right\}, A_{i} \subset \mathbb{Z}$, $i=1,2$, then

$$
\left|\operatorname{Cov}\left(Y_{1}, Y_{2}\right)\right| \leq 8\left(\mathrm{E}\left|Y_{1}\right|^{p_{1}}\right)^{1 / p_{1}}\left(\mathrm{E}\left|Y_{2}\right|^{p_{2}}\right)^{1 / p_{2}} \alpha\left(\operatorname{dis}\left(A_{1}, A_{2}\right)\right)^{1 / p_{3}}
$$

\footnotetext{
${ }^{1}$ Doukhan, P. (1994). Mixing: properties and examples. Lecture Notes in Statistics 85. SpringerVerlag, New York.

${ }^{2}$ Billingsley, P. (1968). Convergence of probability measures. Wiley, New York.
}

imsart-bj ver. 2011/12/06 file: BEJ511.supplementary.material.tex date: February 8, 2013 
where $p_{i}>0, \sum_{i=1}^{3} 1 / p_{i}=1, \operatorname{dis}\left(A_{1}, A_{2}\right)=\min _{a_{1} \in A_{1}, a_{2} \in A_{2}}\left|a_{1}-a_{2}\right|$.

(iii) For random variables $\xi_{1}, \ldots, \xi_{k}, k \geq 1$, let $S_{j}=\sum_{i=0}^{j} \xi_{i}, 0 \leq j \leq k$ and $M_{k}=$ $\max _{0 \leq j \leq k}\left|S_{j}\right|$ (with $\xi_{0} \equiv 0$ ). For some $C_{0}>0, \gamma \geq 0$ and $\beta>1$, if $\mathrm{E}\left[\left|S_{i}-S_{j}\right|^{\gamma}\right] \leq$ $C_{0}(j-i)^{\beta}$ holds for $0 \leq i \leq j \leq k$, then, for all $\lambda>0$,

$$
P\left(M_{k}>\lambda\right) \leq C_{0} 2^{\gamma}\left(1+\left[2^{-1 /(1+\gamma)}-2^{-\beta /(1+\gamma)}\right]^{-\gamma-1}\right) \frac{k^{\beta}}{\lambda^{\gamma}} .
$$

Theorem 5 below gives a bias and variance decomposition for the MBB variance estimator $\hat{\sigma}_{m}^{2}(b)$, uniformly in $b \in \mathcal{J}_{m}$.

Theorem 5. Under the assumptions of Theorem 1, as $m \rightarrow \infty$,

$$
\begin{aligned}
& \text { (i) } \max _{b \in \mathcal{J}_{m}}\left|\left[\operatorname{E} \hat{\sigma}_{m}^{2}(b)-\sigma_{\infty}^{2}\right]+\left(\frac{B_{0}}{b}+\frac{b}{m} \sigma_{\infty}^{2}\right)\right|=O\left(m^{-1}\right) \\
& \text { (ii) } \max _{b \in \mathcal{J}_{m}}\left|\operatorname{Var}\left[\hat{\sigma}_{m}^{2}(b)\right]-V_{0} \frac{b}{m}\right|=O\left(m^{-4 / 3}\right)
\end{aligned}
$$

Proof of Theorem 5(i). Using conditions $D, M_{r=3+a_{0}}$, with independent block resampling, it can be checked that

$$
\begin{aligned}
& m_{1} b \operatorname{Var}_{*}\left(C_{m}^{*}\right) \\
\leq & C b m_{1}^{-2}\left(\left\{1+\left\|\hat{\mu}_{m}\right\|^{2 a_{0}}\right\} \mathrm{E}_{*}\left\|\bar{X}_{1, b}^{*}-\hat{\mu}_{m}\right\|^{6}+m^{-a_{0}} \mathrm{E}_{*}\left\|\bar{X}_{1, b}^{*}-\hat{\mu}_{m}\right\|^{6+2 a_{0}}\right) .
\end{aligned}
$$

Since $\mathrm{E}_{*}\left\|\bar{X}_{1, b}^{*}-\hat{\mu}_{m}\right\|^{\tau} \leq \sum_{i=1}^{m-b+1}\left\|\bar{X}_{i, b}\right\|^{\tau} /(m+b-1)+\left\|\hat{\mu}_{m}\right\|^{\tau}$ for any $\tau>0$, we have $m_{1} b \operatorname{EVar}_{*}\left(C_{m}^{*}\right) \leq C m^{-2}$, using $\mathrm{E}\left\|\bar{X}_{i, b}\right\|^{6+2 a_{0}} \leq C b^{-3-a_{0}}$ and $\mathrm{E}\left\|\hat{\mu}_{m}\right\|^{6+2 a_{0}} \leq C m^{-3-a_{0}}$ by Lemma 1(i). By similar arguments and the Cauchy-Schwarz inequality, it holds that $m_{1} b \operatorname{EVar}_{*}\left(Q_{m}^{*}\right), m_{1} b \mathrm{E}\left|\operatorname{Cov}_{*}\left(L_{m}^{*}, C_{m}^{*}\right)\right| \leq C m^{-1}$ and $m_{1} b \mathrm{E}\left|\operatorname{Cov}_{*}\left(Q_{m}^{*}, C_{m}^{*}\right)\right| \leq C m^{-3 / 2}$. Also, by independent block resampling, we have

$$
m_{1} b \operatorname{Cov}_{*}\left(L_{m}^{*}, Q_{m}^{*}\right)=\frac{b}{m_{1}} \sum_{\substack{v, w \in\left(\mathbb{Z}_{+}\right)^{d} \\\|w\|_{1}=1,\|v\|_{1}=2}} \hat{c}_{w} \hat{c}_{v} \mathrm{E}_{*}\left(\bar{X}_{1, b}^{*}-\hat{\mu}_{m}\right)^{w}\left(\bar{X}_{1, b}^{*}-\hat{\mu}_{m}\right)^{v}
$$

where $\left|\mathrm{E}\left[\mathrm{E}_{*}\left(\bar{X}_{1, b}^{*}-\hat{\mu}_{m}\right)^{w}\left(\bar{X}_{1, b}^{*}-\hat{\mu}_{m}\right)^{v}\right]\right|=\left|\mathrm{E}\left(\bar{X}_{1, b}-\hat{\mu}_{m}\right)^{w}\left(\bar{X}_{1, b}-\hat{\mu}_{m}\right)^{v}\right| \leq C b^{-2}$ holds for $v, w \in\left(\mathbb{Z}_{+}\right)^{d}$ with $\|w\|_{1}=1,\|v\|_{1}=2$ by condition $M_{r=3+a_{0}}$ and Lemma 1 ; after applying condition $D$ (i.e., to expand and replace $\hat{c}_{w} \hat{c}_{v}$ with $c_{w} c_{v}$ ), we may conclude $\left|m_{1} b \mathrm{ECov}_{*}\left(L_{m}^{*}, Q_{m}^{*}\right)\right| \leq C m^{-1}$. 
To complete the prove of Theorem 5(i), we consider finally

$$
\begin{aligned}
& m_{1} b \operatorname{Var}_{*}\left(L_{m}^{*}\right) \\
= & \frac{b}{m-b+1} \sum_{i=1}^{m-b+1}\left(\hat{\nabla}^{\prime} \bar{X}_{i, b}\right)^{2}-b\left(\hat{\nabla}^{\prime} \hat{\mu}_{m}\right)^{2} \\
= & \bar{\sigma}_{m}^{2}(b)-b\left(\nabla^{\prime} \hat{\mu}_{m}\right)^{2}+U_{b, m}, \quad \bar{\sigma}_{m}^{2}(b) \equiv \frac{b}{m-b+1} \sum_{i=1}^{m-b+1}\left(\nabla^{\prime} \bar{X}_{i, b}\right)^{2},
\end{aligned}
$$

with a remainder term $U_{b, m}$ defined by the difference. Using condition $D$ and $M_{r=3+a_{0}}$ with Lemma 1, it can be verified that $\left|\mathrm{E} U_{b, m}\right|,\left|b \mathrm{E}\left(\nabla^{\prime} \hat{\mu}_{m}\right)^{2}-b m^{-1} \sigma_{\infty}^{2}\right|, \mid \mathrm{E}\left[\bar{\sigma}_{m}^{2}(b)\right]-\sigma_{\infty}^{2}+$ $b^{-1} B_{0} \mid \leq C m^{-1}$. Theorem 5(i) then follows as the constants $C$ above are independent of $b \in \mathcal{J}_{m}$.

Proof of Theorem 5(ii). We sketch the proof of the MBB variance expansion. It suffices to show that $\operatorname{Var}\left[m_{1} b \operatorname{Var}_{*}\left(L_{m}^{*}\right)\right]=V_{0} b m^{-1}+O_{u}\left(m^{-4 / 3}\right)=O_{u}\left(m^{-2 / 3}\right)$ and that the second moment of any other individual term in (A.1) of order $O_{u}\left(m^{-2}\right)$. Theorem 5(ii) will then follow by the Cauchy-Schwarz inequality. condition $M_{r}$ in the following refers to $r=6+2 a_{0}$.

Excluding $m_{1} b \operatorname{Var}_{*}\left(L_{m}^{*}\right)$, the second moments of most remaining terms in (A.1) can be readily checked as $O_{u}\left(\mathrm{~m}^{-2}\right)$ using condition $M_{r}$, Lemma 1(i) and Holder's inequality (e.g., $\mathrm{E}\left[m_{1} b \operatorname{Var}_{*}\left(C_{m}^{*}\right)\right]^{2} \leq C m^{-4}$ from (A.2)). Considering a more difficult term, we establish $\mathrm{E}\left[m_{1} b \operatorname{Cov}_{*}\left(L_{m}^{*}, Q_{m}^{*}\right)\right]^{2} \leq C m^{-2}$. Define $m_{1} b{\overline{\operatorname{Cov}_{*}}}_{*}\left(L_{m}^{*}, Q_{m}^{*}\right)$ by replacing $\hat{c}_{v} \hat{c}_{w}$ with $c_{v} c_{w}$ in (A.3), so that $\mathrm{E}\left[m_{1} b \operatorname{Cov}_{*}\left(L_{m}^{*}, Q_{m}^{*}\right)-m_{1} b \overline{\mathrm{Cov}}_{*}\left(L_{m}^{*}, Q_{m}^{*}\right)\right]^{2} \leq C m^{-2}$ follows from $\left\|\hat{\mu}_{m}\right\|^{4+2 a_{0}} \leq C m^{-2-a_{0}}$ and $\mathrm{E}\left\|\bar{X}_{1, b}\right\|^{6} \leq C b^{-3}$ by Lemma 1(i). With Lemma 1(i-ii) and condition $M_{r}$, main components of $\operatorname{Var}\left[m_{1} b \overline{\operatorname{Cov}}_{*}\left(L_{m}^{*}, Q_{m}^{*}\right)\right]$ are bounded as

$$
\begin{aligned}
& \frac{b^{2}}{m_{1}^{2}} \operatorname{Var}\left[\mathrm{E}_{*}\left(\bar{X}_{1, b}^{*}\right)^{w}\left(\bar{X}_{1, b}^{*}\right)^{v}\right] \\
\leq & C \frac{b^{3}}{m_{1}^{2} m} \mathrm{E}\left\|\bar{X}_{1, b}\right\|^{6}+C \frac{b^{2}}{m_{1}^{2} m} \sum_{i=b}^{\infty}\left|\operatorname{Cov}\left[\bar{X}_{1, b}^{w} \bar{X}_{1, b}^{v}, \bar{X}_{1+i, b}^{w} \bar{X}_{1+i, b}^{v}\right]\right| \leq C m^{-2}
\end{aligned}
$$

for $v, w \in\left(\mathbb{Z}_{+}\right)^{d},\|w\|_{1}=1,\|v\|_{1}=2$. Since $\left|\operatorname{Em}_{1} b \overline{\operatorname{Cov}}_{*}\left(L_{m}^{*}, Q_{m}^{*}\right)\right| \leq C m^{-1}$ from the proof of Theorem $5(\mathrm{i}), \mathrm{E}\left[m_{1} b \overline{\operatorname{Cov}}_{*}\left(L_{m}^{*}, Q_{m}^{*}\right)\right]^{2} \leq C m^{-2}$ then follows.

We now consider showing $\operatorname{Var}\left[m_{1} b \operatorname{Var}_{*}\left(L_{m}^{*}\right)\right]=V_{0} b m^{-1}+O_{u}\left(m^{-4 / 3}\right)$, recalling (A.4). Using conditions $D$ and $M_{r}$ with Lemma 1 , it may be shown that $\operatorname{Var}\left[b\left(\hat{\nabla}^{\prime} \hat{\mu}_{m}\right)^{2}\right] \leq$ $C b^{2} / m^{2}$ and

$$
\left|\frac{b^{2}}{m-b+1} \sum_{i=1}^{m-b+1} \operatorname{Cov}\left[\left(\hat{\nabla}^{\prime} \bar{X}_{i, m}\right)^{2},\left(\hat{\nabla}^{\prime} \hat{\mu}_{m}\right)^{2}\right]\right| \leq C b^{2} / m^{2}=O_{u}\left(m^{-4 / 3}\right) .
$$

Expanding $\hat{\nabla}=\left(\partial H\left(\hat{\mu}_{m}\right) / \partial x_{1}, \ldots, \partial H\left(\hat{\mu}_{m}\right) / \partial x_{d}\right)^{\prime}$ around $\mathrm{E} X_{t}=\mu=0$ by Taylor's 
theorem, we have $\hat{\nabla}^{\prime} \bar{X}_{i, b}=\sum_{j=1}^{3} D_{j, i, b}, i=1, \ldots, m-b+1$, where

$$
D_{1, i, b}=\sum_{\substack{w \in\left(\mathbb{Z}_{+}\right)^{d} \\\|w\|_{1}=1}} c_{w} \bar{X}_{i, b}^{w}, \quad D_{2, i, b}=\sum_{\substack{\left.v, w \in \mathbb{Z}_{+}\right)^{d} \\\|w\|_{1}=1,\|v\|_{1}=1}}(w+v) ! c_{v+w} \bar{X}_{i, b}^{w} \hat{\mu}_{m}^{v}
$$

and $D_{3, i, b}$ is a remainder term. From this and recalling $\bar{\sigma}_{m}^{2}(b)$ in (A.4), it can be shown that

$$
\left|\operatorname{Var}\left(\frac{b}{m-b+1} \sum_{i=1}^{m-b+1}\left(\hat{\nabla}^{\prime} \bar{X}_{i, b}\right)^{2}\right)-\operatorname{Var}\left[\bar{\sigma}_{m}^{2}(b)\right]\right| \leq C m^{-4 / 3}
$$

For example, in the differences of variances above, one main component appearing is $\operatorname{Cov}_{m} \equiv b^{2} \sum_{j, i=1}^{m-b+1} \operatorname{Cov}\left[D_{1, i, b}^{2}, D_{1, j, b} D_{2, j, b}\right] /(m-b+1)^{2}$, which in turn is a linear combination of sums of smaller covariance components $b^{2} \sum_{j, i=1}^{m-b+1} \operatorname{Cov}\left[\bar{X}_{i, b}^{v_{1}} \bar{X}_{i, b}^{v_{2}}, \bar{X}_{j, b}^{v_{3}} \bar{X}_{j, b}^{v_{4}} \hat{\mu}_{m}^{v_{5}}\right] /(m-$ $b+1)^{2}$, for $v_{i} \in\left(\mathbb{Z}_{+}\right)^{d},\left\|v_{i}\right\|_{1}=1, i=1, \ldots, 5$. We may rewrite any term $\operatorname{Cov}\left[\bar{X}_{i, b}^{v_{1}} \bar{X}_{i, b}^{v_{2}}, \bar{X}_{j, b}^{v_{3}} \bar{X}_{j, b}^{v_{4}} \hat{\mu}_{m}^{v_{5}}\right]$ as a sum of various products of cumulants $\mathrm{cu}(\cdot)$, one of the most difficult to treat being $\operatorname{Cov}\left[\bar{X}_{j, b}^{v_{3}}, \bar{X}_{j, b}^{v_{4}}\right] \operatorname{cu}\left(\bar{X}_{i, b}^{v_{1}}, \bar{X}_{i, b}^{v_{2}}, \hat{\mu}_{m}^{v_{5}}\right)$ for which we bound

$$
\begin{aligned}
& \frac{b^{2}}{(m-b+1)^{2}} \sum_{j, i=1}^{m-b+1}\left|\operatorname{Cov}\left[\bar{X}_{j, b}^{v_{3}}, \bar{X}_{j, b}^{v_{4}}\right] \operatorname{cu}\left(\bar{X}_{i, b}^{v_{1}}, \bar{X}_{i, b}^{v_{2}}, \hat{\mu}_{m}^{v_{5}}\right)\right| \\
\leq & C \frac{b}{m} \sum_{j=-(m-b)}^{m-b}\left|\operatorname{cu}\left(\bar{X}_{0, b}^{v_{1}}, \bar{X}_{0, b}^{v_{2}}, \bar{X}_{j, b}^{v_{5}}\right)\right| \leq C m^{-4 / 3},
\end{aligned}
$$

recalling $\hat{\mu}_{m}=\sum_{i=1}^{m-b+1} \bar{X}_{i, b} /(m-b+1)$ and using $\left|\operatorname{Cov}\left[\bar{X}_{j, b}^{v_{3}}, \bar{X}_{j, b}^{v_{4}}\right]\right| \leq C b^{-1}$,

$$
b^{2} \sum_{j=-(m-b)}^{m-b}\left|\mathrm{cu}\left(\bar{X}_{0, b}^{v_{1}}, \bar{X}_{0, b}^{v_{2}}, \bar{X}_{j, b}^{v_{5}}\right)\right| \leq C \sum_{s, t \in \mathbb{Z}}(1+|s|+|t|)\left|\mathrm{cu}\left(X_{0}^{v_{1}}, X_{s}^{v_{2}}, X_{t}^{v_{5}}\right)\right|<\infty
$$

by condition $M_{r}$ and Lemma 1(ii). In this fashion, we may ultimately show that $\left|\operatorname{Cov}_{m}\right| \leq$ $\mathrm{Cm}^{-4 / 3}$ in particular and establish (A.5) in general. At this stage, Theorem 5(ii) will now follow by showing $\operatorname{Var}\left[\bar{\sigma}_{m}^{2}(b)\right]=V_{0} b m^{-1}+O_{u}\left(m^{-4 / 3}\right), b \in \mathcal{J}_{m}$, as considered next.

For simplicity, set $Y_{j}=\nabla^{\prime} X_{j}$, let $\bar{Y}_{i, b}=\sum_{j=i}^{b+i-1} Y_{j} / b=\nabla^{\prime} \bar{X}_{i, b}, i=1, \ldots, m-b+1$ and write $\bar{\sigma}_{m}^{2}(b) \equiv b \sum_{i=1}^{m-b+1} \bar{Y}_{i, b}^{2} /(m-b+1)$ in (A.4). Using Lemma 1(i-ii) and condition $M_{r}$, we may write

$$
\begin{aligned}
& \operatorname{Var}\left[\bar{\sigma}_{m}^{2}(b)\right] \\
= & \frac{b^{2} \operatorname{Var}\left(\bar{Y}_{1, b}^{2}\right)}{m-b+1}+\frac{2 b^{2}}{(m-b+1)^{2}} \sum_{i=1}^{m-b}[m-b+1-i] \operatorname{Cov}\left[\bar{Y}_{1, b}^{2}, \bar{Y}_{1+i, b}^{2}\right] \\
= & \frac{2 b^{2}\left[\operatorname{Var}\left(\bar{Y}_{1, b}\right)\right]^{2}}{m-b+1}+\frac{4 b^{2}}{m-b+1} \sum_{i=1}^{b-1}\left[\operatorname{Cov}\left(\bar{Y}_{1, b}, \bar{Y}_{1+i, b}\right)\right]^{2}+O_{u}\left(m^{-4 / 3}\right),
\end{aligned}
$$

imsart-bj ver. 2011/12/06 file: BEJ511.supplementary.material.tex date: February 8, 2013 
using that $\operatorname{Cov}\left[\bar{Y}_{1, b}^{2}, \bar{Y}_{1+i, b}^{2}\right]=2\left[\operatorname{Cov}\left(\bar{Y}_{1, b}, \bar{Y}_{1+i, b}\right)\right]^{2}+\mathrm{cu}\left(\bar{Y}_{1, b}, \bar{Y}_{1, b}, \bar{Y}_{1+i, b}, \bar{Y}_{1+i, b}\right)$ along with $b^{3} \sum_{i=0}^{2 b}\left|\mathrm{cu}\left(\bar{Y}_{1, b}, \bar{Y}_{1, b}, \bar{Y}_{1+i, b}, \bar{Y}_{1+i, b}\right)\right| \leq C \sum_{r, s, t \in \mathbb{Z}}(1+|s|+|t|+|r|)\left|\operatorname{cu}\left(Y_{0}, Y_{r}, Y_{s}, Y_{t}\right)\right|<\infty$ and $\max _{b \leq i \leq 2 b} b^{2}\left|\operatorname{Cov}\left(\bar{Y}_{1, b}, \bar{Y}_{1+i, b}\right)\right| \leq \sum_{k=1}^{\infty} k|r(k)|<\infty$ by condition $M_{r}$. For $i=$ $1, \ldots, b-1$, we use $r(k)=\operatorname{Cov}\left(Y_{0}, Y_{k}\right), k \geq 0$ and $\sigma_{\infty}^{2}=\sum_{k=-\infty}^{\infty} r(k)$ to expand $b^{2} \operatorname{Cov}\left(\bar{Y}_{1, b}, \bar{Y}_{1+i, b}\right)=\sum_{j=1}^{3} P_{j, i, b}$ using terms

$$
P_{1, i, b} \equiv \operatorname{Var}\left(\sum_{j=i+1}^{b} Y_{j}\right)=(b-i) \sigma_{\infty}^{2}-\sum_{k=-\infty}^{\infty}|k| r(k)+R_{1, i, b}
$$

with a remainder such that $\left|R_{1, i, b}\right| \leq 4 \sum_{k=b-i}^{\infty} k|r(k)| \equiv E_{i, b} ; P_{2, i, b} \equiv$

$$
\operatorname{Cov}\left(\sum_{j=1}^{i} Y_{i}, \sum_{j=i+1}^{b} Y_{j}\right)+\operatorname{Cov}\left(\sum_{j=i+1}^{b} Y_{j}, \sum_{j=b+1}^{b+i} Y_{j}\right)=\sum_{k=-\infty}^{\infty}|k| r(k)+R_{2, i, b}
$$

with a remainder such that $\left|R_{2, i, b}\right| \leq E_{i, b}$ if $i>b / 2$ and $\left|R_{2, i, b}\right| \leq \sum_{k=i+1}^{b-i}(k-i)|r(k)|+$ $E_{i, b}$ if $i \leq b / 2$; and $P_{3, i, b} \equiv \operatorname{Cov}\left[\sum_{j=1}^{i} Y_{i}, \sum_{j=b+1}^{b+i} Y_{j}\right]=\sum_{k=-i+1}^{i-1}(i-|k|) r(k+b)$, where $\left|P_{3, i, b}\right| \leq E_{i, b}$. Then, for $i=1, \ldots, b-1$, we have $b^{2} \operatorname{Cov}\left(\bar{Y}_{1, b}, \bar{Y}_{1+i, b}\right)=(b-i) \sigma_{\infty}^{2}+R_{1, i, b}+$ $R_{2, i, b}+P_{3, i, b}$ so that

$$
\left|b^{2} \sum_{i=1}^{b-1}\left[\operatorname{Cov}\left(\bar{Y}_{1, b}, \bar{Y}_{1+i, b}\right)\right]^{2}-\left(3^{-1} b-2^{-1}\right) \sigma_{\infty}^{4}\right| \leq C b^{-1}
$$

using above that $b^{-1} \sum_{i=1}^{b / 2}(b-i) \sum_{k=i+1}^{b-i}(k-i)|r(k)| \leq \sum_{k=1}^{\infty} k|r(k)|<\infty$ and that $\max _{1 \leq i \leq b-1}\left[(b-i) E_{i, b}+\sum_{k=i+1}^{b-i}(k-i)|r(k)|\right] \leq C \sum_{k=1}^{\infty} k^{2}|r(k)|<\infty$ by condition $M_{r}$ along with $b^{-2} \sum_{i=1}^{b-1} i^{2}=3^{-1} b-2^{-1}+6^{-1} b^{-1}$. From this, $\left|m^{-1}-(m-b+1)^{-1}\right|=$ $O_{u}\left(m^{-5 / 3}\right), b \operatorname{Var}\left(\bar{Y}_{1, m}\right)=\sigma_{\infty}^{2}+O_{u}\left(m^{-1 / 3}\right)$ and (A.6), we have finally that

$$
\operatorname{Var}\left[\hat{\sigma}_{m}^{2}(b)\right]=V_{0} \frac{b}{m}+O_{u}\left(m^{-4 / 3}\right), \quad V_{0} \equiv \frac{4}{3} \sigma_{\infty}^{4},
$$

establishing Theorem 5(ii).

Lemma 2 entails that the difference between the expected value $\mathrm{E}\left[\widehat{\mathrm{MSE}}_{m}(b)\right]$ of the HHJ subsample-MSE and its target $\operatorname{MSE}_{m}(b)$ has contributions from the $\operatorname{MSE}_{n}\left(\tilde{\ell}_{n}\right)$ of the pilot MBB estimator $\hat{\sigma}_{n}^{2}\left(\tilde{\ell}_{n}\right)$. A product of bias terms $\mathrm{E}\left[\hat{\sigma}_{n}^{2}\left(\tilde{\ell}_{n}\right)-\sigma_{\infty}^{2}\right] \approx-B_{0} \tilde{\ell}_{n}^{-1}$ and $\mathrm{E}\left[\sum_{i=1}^{n-m+1} \hat{\sigma}_{i, m}^{2}(b) /(n-m+1)-\sigma_{\infty}^{2}\right]=\mathrm{E}\left[\hat{\sigma}_{i, m}^{2}(b)-\sigma_{\infty}^{2}\right] \approx-B_{0} b^{-1}-\sigma_{\infty}^{2} b m^{-1}$ (cf. Theorem 5(i)) also contributes to the expansion in Lemma 2.

Lemma 2. Suppose conditions $D, M_{r}$ with $r=6+2 a_{0}$, and $S$ hold, where $a_{0}$ is as specified by condition $D$. If $m^{-1}+\tilde{\ell}_{n}^{-1}+\tilde{\ell}_{n}^{2} / n \rightarrow 0$ as $n \rightarrow \infty$, then

$$
\begin{aligned}
& \max _{b \in \mathcal{J}_{m}}\left|\mathrm{E}\left[\widehat{\operatorname{MSE}}_{m}(b)\right]-\left[\operatorname{MSE}_{m}(b)+\operatorname{MSE}_{n}\left(\tilde{\ell}_{n}\right)-2 \frac{B_{0}}{\tilde{\ell}_{n}}\left(\frac{B_{0}}{b}+\sigma_{\infty}^{2} \frac{b}{m}\right)\right]\right| \\
= & O\left(n^{-1} m^{2 / 3}+m^{-1} \tilde{\ell}_{n}^{-1}+m^{-1}\left(\tilde{\ell}_{n} / n\right)^{1 / 2}+m^{-1 / 3}\left(\tilde{\ell}_{n} / n\right)\right) .
\end{aligned}
$$

imsart-bj ver. 2011/12/06 file: BEJ511.supplementary.material.tex date: February 8, 2013 
Proof of Lemma 2. For $b \in \mathcal{J}_{m}$, write

$$
\widehat{\operatorname{MSE}}_{m}(b)=\widehat{\operatorname{MSE}}_{m}^{\infty}(b)+\left[\hat{\sigma}_{n}^{2}\left(\tilde{\ell}_{n}\right)-\sigma_{\infty}^{2}\right]^{2}-\frac{2\left[\hat{\sigma}_{n}^{2}\left(\tilde{\ell}_{n}\right)-\sigma_{\infty}^{2}\right]}{n-m+1} \sum_{i=1}^{n-m+1}\left[\hat{\sigma}_{i, m}^{2}(b)-\sigma_{\infty}^{2}\right],
$$

so that taking expectations, and using $\mathrm{E}\left[\widehat{\operatorname{MSE}}_{m}^{\infty}(b)\right]=\operatorname{MSE}_{m}(b)$, yields

$$
\mathrm{E}\left[\widehat{\operatorname{MSE}}_{m}(b)\right]=\operatorname{MSE}_{m}(b)+\operatorname{MSE}_{n}\left(\tilde{\ell}_{n}\right)-2 t_{n}-2 \mathrm{E}\left[\hat{\sigma}_{n}^{2}\left(\tilde{\ell}_{n}\right)-\sigma_{\infty}^{2}\right] \mathrm{E}\left[\hat{\sigma}_{1, m}^{2}(b)-\sigma_{\infty}^{2}\right] .
$$

for $t_{n} \equiv(n-m+1)^{-1} \sum_{i=1}^{n-m+1} \operatorname{Cov}\left[\hat{\sigma}_{n}^{2}\left(\tilde{\ell}_{n}\right), \hat{\sigma}_{i, m}^{2}(b)\right]$. By arguments in Theorem 5(i), the bias $\mathrm{E}\left[\hat{\sigma}_{n}^{2}\left(\tilde{\ell}_{n}\right)-\sigma_{\infty}^{2}\right]=-\tilde{\ell}_{n}^{-1} B_{0}+O(\tilde{\ell} / n)$ so that

$$
\mathrm{E}\left[\hat{\sigma}_{n}^{2}\left(\tilde{\ell}_{n}\right)-\sigma_{\infty}^{2}\right] \mathrm{E}\left[\hat{\sigma}_{1, m}^{2}(b)-\sigma_{\infty}^{2}\right]=\frac{B_{0}}{\tilde{\ell}_{n}}\left(\frac{B_{0}}{b}+\sigma_{\infty}^{2} \frac{b}{m}\right)+O\left(\frac{\tilde{\ell}_{n}}{m^{1 / 3} n}+\frac{1}{m \tilde{\ell}_{n}}\right)
$$

by Theorem 5(i). Lemma 2 then follows by bounding $t_{n}$. If we expand $\hat{\sigma}_{n}^{2}\left(\tilde{\ell}_{n}\right)-\bar{\sigma}_{n}^{2}\left(\tilde{\ell}_{n}\right)$ as a sum of terms analogously to (A.1) and (A.4) (with respect to a size $n$ sample), then any term in this sum has a variance of order $O\left[\left(\tilde{\ell}_{n} / n\right)^{2}\right]$ since $\tilde{\ell}_{n}^{2} / n \rightarrow 0$ [cf. proof of Theorem 5(ii)] while $\operatorname{Var}\left[\hat{\sigma}_{1, m}^{2}(b)\right] \leq C m^{-2 / 3}$ by Theorem 5(ii). By the Cauchy-Schwarz inequality,

$$
\left|t_{n}\right| \leq\left|t_{1, n}\right|+O\left(m^{-1 / 3} \tilde{\ell}_{n} / n\right), \quad t_{1, n} \equiv \frac{1}{n-m+1} \sum_{i=1}^{n-m+1} \operatorname{Cov}\left[\bar{\sigma}_{n}^{2}\left(\tilde{\ell}_{n}\right), \hat{\sigma}_{i, m}^{2}(b)\right] .
$$

If we write the difference $\left\{\hat{\sigma}_{i, m}^{2}(b)-\left[\bar{\sigma}_{i, m}^{2}(b)-b\left(\nabla^{\prime} \hat{\mu}_{i, m}\right)^{2}+2 L Q_{i, m}^{*}\right]\right\}$ [where here $L Q_{i, m}^{*} \equiv$ $\left.b m_{1}^{-1} \sum_{\|w\|_{1}=1,\|v\|_{1}=2} c_{w} c_{v} \mathrm{E}_{*}\left(\bar{X}_{1, b}^{*}-\hat{\mu}_{m}\right)^{w}\left(\bar{X}_{1, b}^{*}-\hat{\mu}_{m}\right)^{v}\right]$ as a sum of terms using (A.1), (A.3), (A.4) and condition $D$, then any term in this sum has a variance of order $O\left(\mathrm{~m}^{-2}\right)$, while $\operatorname{Var}\left[\bar{\sigma}_{n}^{2}\left(\tilde{\ell}_{n}\right)\right] \leq C \tilde{\ell}_{n} / n$ (analogously to the main variance component in Theorem 5(ii)). Again by the Cauchy-Schwarz inequality, $\left|t_{1, n}\right| \leq\left|t_{2, n}\right|+O\left(m^{-1}(\tilde{\ell} / n)^{1 / 2}\right)$, for

$$
t_{2, n} \equiv \frac{1}{n-m+1} \sum_{i=1}^{n-m+1} \operatorname{Cov}\left[\bar{\sigma}_{n}^{2}\left(\tilde{\ell}_{n}\right), \bar{\sigma}_{i, m}^{2}(b)-b\left(\nabla^{\prime} \hat{\mu}_{i, m}\right)^{2}+2 L Q_{i, m}^{*}\right] .
$$

Finally, it can be shown $\left|t_{2, n}\right| \leq C m^{-1 / 3} n^{-1}\left(m+\tilde{\ell}_{n}\right)$, uniformly in $b \in \mathcal{J}_{m}$. For example, the contribution $t_{3, n} \equiv(n-m+1)^{-1} \sum_{i=1}^{n-m+1} \operatorname{Cov}\left[\bar{\sigma}_{n}^{2}\left(\tilde{\ell}_{n}\right), \bar{\sigma}_{i, m}^{2}(b)\right]$ to $t_{2, n}$ is bounded as

$$
\begin{aligned}
\left|t_{3, n}\right| & \leq C \frac{\tilde{\ell}_{n}}{n} \sum_{i=-n}^{n}\left|\operatorname{Cov}\left[\left(\nabla^{\prime} \bar{X}_{1, \tilde{\ell}_{n}}\right)^{2}, \bar{\sigma}_{1+i, m}^{2}(b)\right]\right| \\
& \leq C \frac{\tilde{\ell}_{n} \max \left\{m, \tilde{\ell}_{n}\right\}}{n}\left\{\operatorname{Var}\left[\left(\nabla^{\prime} \bar{X}_{1, \tilde{\ell}_{n}}\right)^{2}\right] \operatorname{Var}\left[\bar{\sigma}_{1, m}^{2}(b)\right]\right\}^{1 / 2}+\frac{C}{n} \\
& \leq C \frac{\max \left\{m, \tilde{\ell}_{n}\right\}}{n m^{1 / 3}},
\end{aligned}
$$

imsart-bj ver. 2011/12/06 file: BEJ511.supplementary.material.tex date: February 8, 2013 
using Theorem 5(ii) with $\tilde{\ell}_{n} \sum_{|i|>2 \max \left\{m, \tilde{\ell}_{n}\right\}}\left|\operatorname{Cov}\left[\left(\nabla^{\prime} \bar{X}_{1, \tilde{\ell}_{n}}\right)^{2}, \bar{\sigma}_{1+i, m}^{2}(b)\right]\right| \leq C$ by Lemma 1(iii) and condition $M_{r}, r=6+2 a_{0}$.

To state Lemma 3 below, used in the proof of Theorem 6 of Section A.3, we require some further notation.

For $b \in \mathcal{J}_{m}$, recall the definition $\bar{\sigma}_{m}^{2}(b) \equiv b \sum_{i=1}^{m-b+1} \bar{Y}_{i, b}^{2} /(m-b+1)$, where $\bar{Y}_{i, b}=$ $\sum_{j=i}^{b+i-1} Y_{j} / b=\nabla^{\prime} \bar{X}_{i, b}, i=1, \ldots, m-b+1$ (i.e., $Y_{j}=\nabla^{\prime} X_{j}$ ) in (A.4); we continue to assume $\mathrm{E} X_{t}=\mu=0$. Let $\bar{\sigma}_{i, m}^{2}(b)$ denote the version of $\bar{\sigma}_{m}^{2}(b)$ defined on subsample $\mathcal{X}_{i, m}$ and decompose $\left[\hat{\sigma}_{i, m}^{2}(b)-\sigma_{\infty}^{2}\right]^{2}=\left[\bar{\sigma}_{i, m}^{2}(b)-\sigma_{\infty}^{2}\right]^{2}+R_{i, m}(b)$ involving a remainder $R_{i, m}(b), i=1, \ldots, n-m+1$. Recalling

$$
\widehat{\operatorname{MSE}}_{m}^{\infty}(b)=\frac{1}{n-m+1} \sum_{i=1}^{n-m+1}\left[\hat{\sigma}_{i, m}^{2}(b)-\sigma_{\infty}^{2}\right]^{2}
$$

from (2.8) of the main manuscript, write $\widehat{\operatorname{MSE}}_{m}^{\infty}(b)=\widehat{\operatorname{MSE}}_{m}^{(1), \infty}(b)+\widehat{\operatorname{MSE}}_{m}^{(2), \infty}(b)$ for $b \in \mathcal{J}_{m}$, where $\widehat{\mathrm{MSE}}_{m}^{(1), \infty}(b) \equiv \sum_{i=1}^{n-m+1}\left[\bar{\sigma}_{i, m}^{2}(b)-\sigma_{\infty}^{2}\right]^{2} /(n-m+1)$ and $\widehat{\mathrm{MSE}}_{m}^{(2), \infty}(b) \equiv$ $\sum_{i=1}^{n-m+1} R_{i, m}(b) /(n-m+1)$. Let $\Delta_{m}^{(1), \infty}(b)$ and $\Delta_{m}^{(2), \infty}(b)$ denote the analogs of

$$
\Delta_{m}^{\infty}(b)=\left\{\widehat{\operatorname{MSE}}_{m}^{\infty}\left(b_{m}^{\text {opt }}\right)-\mathrm{E}\left[\widehat{\operatorname{MSE}}_{m}^{\infty}\left(b_{m}^{\text {opt }}\right)\right]\right\}-\left\{\widehat{\operatorname{MSE}}_{m}^{\infty}(b)-\mathrm{E}\left[\widehat{\operatorname{MSE}}_{m}^{\infty}(b)\right]\right\}
$$

(given in (4.1) of the main manuscript) defined with respect to $\widehat{\mathrm{MSE}}_{m}^{(1), \infty}(b)$ and $\widehat{\mathrm{MSE}}_{m}^{(2), \infty}(b)$ (rather than $\left.\widehat{\operatorname{MSE}}_{m}^{\infty}(b)\right)$, so that

$$
\Delta_{m}^{\infty}(b)=\Delta_{m}^{(1), \infty}(b)+\Delta_{m}^{(2), \infty}(b), b \in \mathcal{J}_{m} .
$$

Next define $\Delta_{m}(b)$ by replacing $\widehat{\operatorname{MSE}}_{m}^{\infty}(\cdot)$ from (A.7) with

$$
\widehat{\operatorname{MSE}}_{m}(b)=\frac{1}{n-m+1} \sum_{i=1}^{n-m+1}\left[\hat{\sigma}_{i, m}^{2}(b)-\hat{\sigma}_{n}^{2}\left(\tilde{\ell}_{n}\right)\right]^{2}
$$

(given in (2.7) of the main manuscript) in (A.8) (i.e., the HHJ method uses $\widehat{\mathrm{MSE}}_{m}$ ). Then, for any $b \in \mathcal{J}_{m}$,

$$
\Delta_{m}(b)=\Delta_{m}^{\infty}(b)+\Omega_{1, m}(b)+\Omega_{2, m}(b), \Omega_{1, m}(b) \equiv \Omega_{3, m}(b)-\mathrm{E}\left[\Omega_{3, m}(b)\right]
$$

holds, for $\Omega_{2, m}(b) \equiv 2\left[\hat{\sigma}_{n}^{2}\left(\tilde{\ell}_{n}\right)-\mathrm{E} \hat{\sigma}_{n}^{2}\left(\tilde{\ell}_{n}\right)\right] \mathrm{E}\left[\hat{\sigma}_{1, m}^{2}\left(b_{m}^{\text {opt }}\right)-\hat{\sigma}_{1, m}^{2}(b)\right]$ and

$$
\Omega_{3, m}(b) \equiv 2 \frac{\hat{\sigma}_{n}^{2}\left(\tilde{\ell}_{n}\right)-\sigma_{\infty}^{2}}{n-m+1} \sum_{i=1}^{n-m+1}\left\{\left[\hat{\sigma}_{i, m}^{2}(b)-\hat{\sigma}_{i, m}^{2}\left(b_{m}^{\mathrm{opt}}\right)\right]-\mathrm{E}\left[\hat{\sigma}_{i, m}^{2}(b)-\hat{\sigma}_{i, m}^{2}\left(b_{m}^{\mathrm{opt}}\right)\right]\right\} .
$$


Lemma 3. Under the assumptions of Theorem 6 (for which $r=14+4 a_{0}$ and $\delta>0$ in condition $M_{r}$ ), there exists $C_{0}>0$, and integer $N_{0} \geq 1$ such that for all $n \geq N_{0}$ and $b, b_{1} \in \mathcal{J}_{m}:$

(i) $\quad\left(\mathrm{E}\left|R_{1, m}(b)\right|^{\kappa}\right)^{1 / \kappa} \leq C_{0} m^{-1}, \quad \kappa=2\left[1+\frac{\delta}{(2 r+\delta)(2 r-1)-\delta-1}\right]$.
(ii) $\quad \mathrm{E}\left\{\left[\left(\frac{n}{m}\right)^{1 / 2} m^{2 / 3} \Delta_{m}^{(2), \infty}(b)\right]^{2}\right\} \leq C_{0} m^{-2 / 3}$.
(iii) $\quad \mathrm{E}\left\{\left[\left(\frac{n}{m}\right)^{1 / 2} m^{2 / 3}\left[\Delta_{m}^{(1), \infty}(b)-\Delta_{m}^{(1), \infty}\left(b_{1}\right)\right]\right]^{4}\right\} \leq C_{0} m^{-2 / 3}\left(b-b_{1}\right)^{2}$.
(iv) $\quad \mathrm{E}\left\{\left[\left(\frac{n}{m}\right)^{1 / 2} m^{2 / 3} \Omega_{1, m}(b)\right]^{2}\right\} \leq C_{0} m^{-1}$.
(v) $\quad \mathrm{E}\left\{\left[\left(\frac{n}{m}\right)^{1 / 2} m^{2 / 3}\left[\Omega_{2, m}(b)-\Omega_{2, m}\left(b_{1}\right)\right]\right]^{2}\right\} \leq C_{0} \frac{\tilde{\ell}_{n}}{m}\left(b-b_{1}\right)^{2}$.

Proof of Lemma 3. We sketch the proof. Fix $b \in \mathcal{J}_{m}$ and expand $\hat{\sigma}_{1, m}^{2}(b) \equiv \hat{\sigma}_{m}^{2}(b)$ as in (A.1). By construction, the remainder $R_{1, m}(b)$ is a linear combination of terms of the form $A_{m}^{2}(b)$ and $\left[\bar{\sigma}_{1, m}^{2}(b)-\sigma_{\infty}^{2}\right] A_{m}(b)$ where $A_{m}(b)$ is selected from the collection: $\left[m_{1} b \operatorname{Var}_{*}\left(L_{m}^{*}\right)-\bar{\sigma}_{1, m}^{2}(b)\right], m_{1} b \operatorname{Var}_{*}\left(Q_{m}^{*}\right), m_{1} b \operatorname{Var}_{*}\left(C_{m}^{*}\right), m_{1} b \operatorname{Cov}_{*}\left(L_{m}^{*}, Q_{m}^{*}\right), m_{1} b \operatorname{Cov}_{*}\left(L_{m}^{*}, C_{m}^{*}\right)$. Using Lemma 1, it can be shown that

$$
\left\{\mathrm{E}\left[A_{m}^{2 \kappa}(b)\right]\right\}^{1 / \kappa} \leq C m^{-4 / 3},
$$

uniformly in $b \in \mathcal{J}_{m}$. For illustration, $\left\{\mathrm{E}\left[m_{1} b \operatorname{Var}_{*}\left(C_{m}^{*}\right)\right]^{2 \kappa}\right\}^{1 / \kappa} \leq C m^{-2}$ holds, using (A.2) and Holder's inequality to bound moments in terms of powers of $\left(\mathrm{E}\left\|\hat{\mu}_{m}\right\|^{2 r}\right)^{1 /(2 r)} \leq$ $C m^{-1}$ and $\left(\mathrm{E}\left\|\bar{X}_{1, b}\right\|^{2 r}\right)^{1 /(2 r)} \leq C b^{-1}, b \in \mathcal{J}_{m}\left(\right.$ since $\left(12+4 a_{0}\right) \kappa<2 r$ from $\left(12+4 a_{0}\right)[\kappa-$ $2]<1$ ) by Lemma $1(\mathrm{i})$. Similarly, using condition $D$, Lemma 1(i) and (A.3),

$$
\begin{aligned}
& \left\{\mathrm{E}\left[m_{1} b \operatorname{Cov}_{*}\left(L_{m}^{*}, Q_{m}^{*}\right)\right]^{2 \kappa}\right\}^{1 / \kappa} \\
\leq & C b^{2} m_{1}^{-2}\left[\mathrm{E}\left\|\bar{X}_{1, m}\right\|^{6 \kappa}+\left(\mathrm{E}\left\|\bar{X}_{1, m}\right\|^{12 \kappa}\right)^{1 / 2}\left\{\left(\mathrm{E}\left\|\hat{\mu}_{m}\right\|^{4 \kappa}\right)^{1 / 2}+\right.\right. \\
& \left.\left.\left(\mathrm{E}\left\|\hat{\mu}_{m}\right\|^{\left(4+4 a_{0}\right) \kappa}\right)^{1 / 2}\right\}+\mathrm{E}\left\|\hat{\mu}_{m}\right\|^{6 \kappa}+\mathrm{E}\left\|\hat{\mu}_{m}\right\|^{8 \kappa}+\mathrm{E}\left\|\hat{\mu}_{m}\right\|^{\left(8+2 a_{0}\right) \kappa}\right]^{1 / \kappa} \\
\leq & C m^{-5 / 3} .
\end{aligned}
$$

Using (A.4), we may write $\left[m_{1} b \operatorname{Var}_{*}\left(L_{m}^{*}\right)-\bar{\sigma}_{1, m}^{2}(b)\right]=-b\left(\hat{\nabla}^{\prime} \hat{\mu}_{m}\right)^{2}+U_{b, m}$, where it holds that $\left\{\mathrm{E}\left[b\left(\hat{\nabla}^{\prime} \hat{\mu}_{m}\right)^{2}\right]^{2 \kappa}\right\}^{1 / \kappa} \leq C b^{2} / m^{2} \leq C m^{-4 / 3}$ using condition $D$ and Lemma 1(i). It also holds that $\left\{E\left[U_{b, m}^{2 \kappa}\right]\right\}^{1 / \kappa} \leq\left\{E\left[\bar{U}_{b, m}^{6}\right]\right\}^{1 / 3} \leq C m^{-4 / 3}$, based on arguments in the proofs of Theorem 5 showing that $\left|\mathrm{E} U_{b, m}\right| \leq C m^{-1}$ and $\operatorname{Var}\left(U_{b, m}\right) \leq C m^{-4 / 3}$ and using that the 3rd-order cumulants $\left|\mathrm{cu}\left(U_{b, m}, U_{b, m}, U_{b, m}\right)\right| \leq C m^{-2}$ while 4 th and 6th order cumulants of $U_{b, m}$ are bounded by $\mathrm{Cm}^{-8 / 3}$ and $\mathrm{Cm}^{-4}$ (uniformly in $b \in \mathcal{J}$ ) from conditions $D$ and $M_{r}\left(r=14+4 a_{0}\right)$ and Lemma 1(i-ii). 
From (A.11) and the Cauchy-Schwarz inequality, to show $\left\{\mathrm{E}\left|\left[\bar{\sigma}_{1, m}^{2}(b)-\sigma_{\infty}^{2}\right] A_{m}(b)\right|^{\kappa}\right\}^{1 / \kappa}$ contributions to $\left\{\mathrm{E}\left|R_{1, m}(b)^{\kappa}\right|\right\}^{1 / \kappa}$ are bounded by $\mathrm{Cm}^{-1}$ (uniformly in $b \in \mathcal{J}_{m}$ ), it suffices to show

$$
\left\{\mathrm{E}\left[\bar{\sigma}_{1, m}^{2}(b)-\sigma_{\infty}^{2}\right]^{4 \kappa}\right\}^{1 /(4 \kappa)} \leq\left\{\mathrm{E}\left[\bar{\sigma}_{1, m}^{2}(b)-\sigma_{\infty}^{2}\right]^{10}\right\}^{1 / 10} \leq C m^{-1 / 3},
$$

as $10>4 \kappa$. For $s, t, u \in \mathbb{Z}_{+}, 0 \leq s \leq t \leq u$, writing $\operatorname{cu}\left(\bar{Y}_{0, b}^{2}, \bar{Y}_{s, b}^{2}, \bar{Y}_{t, b}^{2}, \bar{Y}_{u, b}^{2}\right)=$

$$
\begin{aligned}
& \mathrm{E}\left[\left(\bar{Y}_{0, b}^{2}-\mathrm{E} \bar{Y}_{0, b}^{2}\right)\left(\bar{Y}_{s, b}^{2}-\mathrm{E} \bar{Y}_{s, b}^{2}\right)\left(\bar{Y}_{t, b}^{2}-\mathrm{E} \bar{Y}_{t, b}^{2}\right)\left(\bar{Y}_{u, b}^{2}-\mathrm{E} \bar{Y}_{u, b}^{2}\right)\right]-\operatorname{Cov}\left(\bar{Y}_{0, b}^{2}, \bar{Y}_{s, b}^{2}\right) \times \\
& \operatorname{Cov}\left(\bar{Y}_{t, b}^{2}, \bar{Y}_{u, b}^{2}\right)-\operatorname{Cov}\left(\bar{Y}_{0, b}^{2}, \bar{Y}_{t, b}^{2}\right) \operatorname{Cov}\left(\bar{Y}_{s, b}^{2}, \bar{Y}_{u, b}^{2}\right)-\operatorname{Cov}\left(\bar{Y}_{0, b}^{2}, \bar{Y}_{u, b}^{2}\right) \operatorname{Cov}\left(\bar{Y}_{s, b}^{2}, \bar{Y}_{t, b}^{2}\right),
\end{aligned}
$$

it holds that $\left|\operatorname{cu}\left(\bar{Y}_{0, b}^{2}, \bar{Y}_{s, b}^{2}, \bar{Y}_{t, b}^{2}, \bar{Y}_{u, b}^{2}\right)\right| \leq C b^{-4}$ by Lemma 1(i) if $d(s, t, u) \equiv \max \{s, t-$ $s, u-t\} \leq 2 b$ and $\left|\mathrm{cu}\left(\bar{Y}_{0, b}^{2}, \bar{Y}_{s, b}^{2}, \bar{Y}_{t, b}^{2}, \bar{Y}_{u, b}^{2}\right)\right| \leq C\left[\mathrm{E}\left(\bar{Y}_{0, b}^{2 \eta}\right)\right]^{4 / \eta} \alpha(d(s, t, u)-b)^{(\eta-4) / \eta} \leq$ $C b^{-4} \alpha(d(s, t, u)-b)^{(\eta-4) / \eta}$ by Lemma 1 (i-ii) if $d(s, t, u)>2 b$, where $\eta>4$ is chosen (e.g., $\eta=5$ ) so that $\eta \leq r$ (i.e., Lemma 1 (ii) applies to $\left.\mathrm{E}\left(\bar{Y}_{0, b}^{2 \eta}\right)\right)$ and $\sum_{k=1}^{\infty} k^{2} \alpha(k)^{(\eta-4) / \eta}<\infty$ under condition $M_{r}$. As the size of the set $\{(s, t, u): 0 \leq s \leq t \leq u, d(s, t, u)=k\}$ is no more than $3(1+3 k)^{2}, k \geq 0$, we may bound

$$
\begin{aligned}
& \left|\mathrm{cu}\left[\bar{\sigma}_{1, m}^{2}(b), \bar{\sigma}_{1, m}^{2}(b), \bar{\sigma}_{1, m}^{2}(b), \bar{\sigma}_{1, m}^{2}(b)\right]\right| \\
\leq & C \frac{b^{4}}{m^{3}} \sum_{\substack{s, t, u \in \mathbb{E}_{+} \\
0 \leq s \leq t \leq u \leq m}}\left|\operatorname{cu}\left(\bar{Y}_{0, b}^{2}, \bar{Y}_{s, b}^{2}, \bar{Y}_{t, b}^{2}, \bar{Y}_{u, b}^{2}\right)\right| \\
\leq & C \frac{b^{3}}{m^{3}}+\frac{C}{m^{3}} \sum_{k=1}^{\infty} k^{2} \alpha(k)^{(\eta-4) / \eta} \leq C m^{-2} .
\end{aligned}
$$

By similar arguments, the $i$ th order cumulant of $\bar{\sigma}_{1, m}^{2}(b)$ is bounded by $C m^{-2(i-1) / 3}$, uniformly in $b \in \mathcal{J}_{m}$, for $i=2, \ldots, 10$. Then, (A.12) follows from these cumulant bounds and $\left|\mathrm{E}\left[\bar{\sigma}_{1, m}^{2}(b)-\sigma_{\infty}^{2}\right]\right| \leq C b^{-1} \leq C m^{-1 / 3}$ and $\operatorname{Var}\left[\bar{\sigma}_{1, m}^{2}(b)\right] \leq C m^{-1} b \leq C m^{-2 / 3}$ (cf. the proof of Theorem 5). Having shown (A.11) and (A.12), Lemma 3(i) now follows.

Since $\mathrm{E}\left[\Delta_{m}^{(2), \infty}(b)\right]=0$, Lemma 3(ii) follows from

$$
\frac{n}{m} m^{4 / 3} \operatorname{Var}\left[\Delta_{m}^{(2), \infty}(b)\right] \leq \sum_{\tilde{b} \in\left\{b, b_{m}^{o p t}\right\}} m^{1 / 3} \sum_{i=-n}^{n}\left|\operatorname{Cov}\left[R_{1, m}(\tilde{b}), R_{1+i, m}(\tilde{b})\right]\right| \leq \frac{C}{m^{2 / 3}}
$$

by Lemma 1(ii), Lemma 3(i) (i.e., $\mathrm{E}\left[R_{1, m}(b)^{2}\right] \leq C m^{-2}$ ) and condition $M_{r}$. In particular, $\sum_{i=0}^{m}\left|\operatorname{Cov}\left[R_{1, m}(b), R_{1+i, m}(b)\right]\right| \leq C m^{-1}$ is used above with $\sum_{i=m+1}^{n}\left|\operatorname{Cov}\left[R_{1, m}(b), R_{1+i, m}(b)\right]\right| \leq$ $\left\{\mathrm{E}\left[R_{1, m}(b)^{\kappa}\right]\right\}^{2 / \kappa} \sum_{i=m+1}^{n} \alpha(i-m)^{(\kappa-2) / \kappa} \leq C m^{-2}$ since $\alpha(k)^{(\kappa-2) / \kappa} \leq C k^{-\tau}$ under condition $M_{r}$ for $\tau \equiv(2 r-1)(2 r+\delta)(\kappa-2) /(\kappa \delta)>2$ by the definition of $\kappa$.

To prove Lemma $3(\mathrm{iii})$, we first show, for $b, b_{1} \in \mathcal{J}_{m}$,

$$
\begin{aligned}
& \mathrm{E}\left\{\left[\left(\frac{n}{m}\right)^{1 / 2} m^{2 / 3}\left[\Delta_{m}^{(1), \infty}(b)-\Delta_{m}^{(1), \infty}\left(b_{1}\right)\right]\right]^{2}\right\} \\
= & m^{4 / 3} \frac{n}{m} \operatorname{Var}\left(\Delta_{m}^{(1), \infty}(b)-\Delta_{m}^{(1), \infty}\left(b_{1}\right)\right) \leq C m^{-1 / 3}\left|b-b_{1}\right|,
\end{aligned}
$$

imsart-bj ver. 2011/12/06 file: BEJ511.supplementary.material.tex date: February 8, 2013 
recalling $\mathrm{E}\left[\Delta_{m}^{(1), \infty}(b)\right]=0$ and that $\Delta_{m}^{(1), \infty}(b)-\Delta_{m}^{(1), \infty}\left(b_{1}\right)=\left[\widehat{\mathrm{MSE}}_{m}^{(1), \infty}(b)-\mathrm{E}\left\{\widehat{\mathrm{MSE}}_{m}^{(1), \infty}(b)\right\}-\right.$ $\left[\widehat{\operatorname{MSE}}_{m}^{(1), \infty}\left(b_{1}\right)-\mathrm{E}\left\{\widehat{\operatorname{MSE}}_{m}^{(1), \infty}\left(b_{1}\right)\right\}\right]$ by definition. Fix $b, b_{1} \in \mathcal{J}_{m}$ with $b>b_{1}$ and define a covariance $c_{b, b_{1}, m}(k)=\operatorname{Cov}\left(\left[\bar{\sigma}_{1, m}^{2}(b)-\sigma_{\infty}^{2}\right]^{2}-\left[\bar{\sigma}_{1, m}^{2}\left(b_{1}\right)-\sigma_{\infty}^{2}\right]^{2},\left[\bar{\sigma}_{k+1, m}^{2}(b)-\sigma_{\infty}^{2}\right]^{2}-\right.$ $\left.\left[\bar{\sigma}_{k+1, m}^{2}\left(b_{1}\right)-\sigma_{\infty}^{2}\right]^{2}\right), k \geq 0$. Then,

$$
\begin{aligned}
& m^{4 / 3} \frac{n}{m} \operatorname{Var}\left(\Delta_{m}^{(1), \infty}(b)-\Delta_{m}^{(1), \infty}\left(b_{1}\right)\right) \\
\leq & C m^{4 / 3} c_{b, b_{1}, m}(0)+m^{4 / 3} \sum_{k=2 m+1}^{\infty}\left|c_{b, b_{1}, m}(k)\right| \\
\leq & C\left[m^{4 / 3} c_{b, b_{1}, m}(0)+m^{-1}\right],
\end{aligned}
$$

using $m^{7 / 3} \sum_{k=2 m+1}^{\infty}\left|c_{b, b_{1}, m}(k)\right| \leq C \sum_{k=1}^{\infty} k \alpha(k)^{(\kappa-2) / \kappa} \leq C$ by Lemma 1 (i-ii), (A.12), and condition $M_{r}$ with $\alpha(k)^{(\kappa-2) / \kappa} \leq C k^{-\tau}, \tau>2$. Then, (A.14) will follow from (A.15) by showing

$$
m^{4 / 3} c_{b, b_{1}, m}(0) \leq C \frac{b-b_{1}}{m^{1 / 3}}, \quad b>b_{1} \in \mathcal{J}_{m},
$$

where $m^{4 / 3} c_{b, b_{1}, m}(0)=-2 m^{4 / 3} A_{b, b_{1}, m}+\sum_{\tilde{b} \in\left\{b, b_{1}\right\}} m^{4 / 3} \operatorname{Var}\left(\left[\bar{\sigma}_{1, m}^{2}(\tilde{b})-\sigma_{\infty}^{2}\right]^{2}\right)$ for $A_{b, b_{1}, m} \equiv$ $\operatorname{Cov}\left(\left[\bar{\sigma}_{1, m}^{2}(b)-\sigma_{\infty}^{2}\right]^{2},\left[\bar{\sigma}_{1, m}^{2}\left(b_{1}\right)-\sigma_{\infty}^{2}\right]^{2}\right)$. By Theorem 5 , (A.13) and $\left|\operatorname{cu}\left[\bar{\sigma}_{1, m}^{2}(b), \bar{\sigma}_{1, m}^{2}(b), \bar{\sigma}_{1, m}^{2}(b)\right]\right| \leq$ $\mathrm{Cm}^{-4 / 3}$ (cf. sentence following (A.13)), we have

$$
\left|m^{4 / 3} \operatorname{Var}\left(\left[\bar{\sigma}_{1, m}^{2}(b)-\sigma_{\infty}^{2}\right]^{2}\right)-2\left(V_{0} \frac{b}{m^{1 / 3}}\right)^{2}\right| \leq C m^{-1 / 3}, \quad b \in \mathcal{J}_{m} .
$$

Similarly to (A.13), it can be shown $\left|\mathrm{cu}\left[\bar{\sigma}_{1, m}^{2}(b), \bar{\sigma}_{1, m}^{2}(b), \bar{\sigma}_{1, m}^{2}\left(b_{1}\right), \bar{\sigma}_{1, m}^{2}\left(b_{1}\right)\right]\right| \leq \mathrm{Cm}^{-2}$ and $\left|\mathrm{cu}\left[\bar{\sigma}_{1, m}^{2}(\tilde{b}), \bar{\sigma}_{1, m}^{2}(b), \bar{\sigma}_{1, m}^{2}\left(b_{1}\right)\right]\right| \leq C m^{-4 / 3}$ for $b, b_{1} \in \mathcal{J}_{m}, \tilde{b}=b$ or $b_{1}$, so that

$$
\left|m^{4 / 3} A_{b, b_{1}, m}-2 m^{4 / 3} B_{b, b_{1}, m}\right| \leq C m^{-1 / 3}
$$

for $B_{b, b_{1}, m} \equiv\left\{\operatorname{Cov}\left[\bar{\sigma}_{1, m}^{2}(b), \bar{\sigma}_{1, m}^{2}\left(b_{1}\right)\right]\right\}^{2}$ by Theorem 5 . Since

$$
\begin{aligned}
& \frac{b b_{1}}{m^{2}} \sum_{i=1}^{m-b+1} \sum_{j=1}^{m-b_{1}+1}\left|\mathrm{cu}\left(\bar{Y}_{i, b}, \bar{Y}_{i, b}, \bar{Y}_{j, b_{1}}, \bar{Y}_{j, b_{1}}\right)\right| \\
\leq & C \frac{b b_{1}}{m} \sum_{j=-m}^{m}\left|\operatorname{cu}\left(\bar{Y}_{0, b}, \bar{Y}_{0, b}, \bar{Y}_{j, b_{1}}, \bar{Y}_{j, b_{1}}\right)\right| \leq C m^{-4 / 3},
\end{aligned}
$$

by bounding $m \sum_{j=-m}^{m}\left|\mathrm{cu}\left(\bar{Y}_{0, b}, \bar{Y}_{0, b}, \bar{Y}_{j, b_{1}}, \bar{Y}_{j, b_{1}}\right)\right|$ by $\sum_{s, t, u \in \mathbb{Z}}(1+|s|+|t|+|u|)\left|\mathrm{cu}\left(Y_{0}, Y_{s}, Y_{t}, Y_{u}\right)\right|<$ $\infty$ from Lemma 1(ii) and condition $M_{r}$, and

$$
\frac{b b_{1}}{m^{2}} \sum_{i=m-b+1}^{m-b_{1}+1} \sum_{j=1}^{m-b_{1}+1}\left[\operatorname{Cov}\left(\bar{Y}_{i, b}, \bar{Y}_{j, b_{1}}\right)\right]^{2} \leq C \frac{\left(b-b_{1}\right)}{m}
$$

imsart-bj ver. 2011/12/06 file: BEJ511.supplementary.material.tex date: February 8, 2013 
by Lemma 1(i) and $b>b_{1}$, we further have

$$
\left|2 m^{4 / 3} B_{b, b_{1}, m}-2 m^{4 / 3} C_{b, b_{1}, m}^{2}\right| \leq C\left[m^{-2 / 3}+m^{-1 / 3}\left(b-b_{1}\right)\right],
$$

for $C_{b, b_{1}, m} \equiv 2 b b_{1} \sum_{i, j=1}^{m-b_{1}+1}\left[\operatorname{Cov}\left(\bar{Y}_{i, b}, \bar{Y}_{j, b_{1}}\right)\right]^{2} /\left(m-b_{1}+1\right)^{2}$. Then, writing $\operatorname{Cov}\left(\bar{Y}_{i, b}, \bar{Y}_{j, b_{1}}\right)=$ $\operatorname{Cov}\left(\left[b_{1} / b\right] \bar{Y}_{i, b_{1}}+b^{-1} S_{i}, \bar{Y}_{j, b_{1}}\right)$ with $S_{i} \equiv \sum_{k=i+b_{1}}^{i+b-1} Y_{k}$ for $i, j=1, \ldots, m-b_{1}+1$ and defining

$$
D_{b, b_{1}, m} \equiv \sum_{j=-m}^{m}\left|\operatorname{Cov}\left[\bar{Y}_{1, b_{1}}, \bar{Y}_{1+j, b_{1}}\right] \operatorname{Cov}\left[\bar{Y}_{1, b_{1}}, b^{-1} S_{1+j}\right]\right|+\left|\operatorname{Cov}\left[\bar{Y}_{1, b_{1}}, b^{-1} S_{1+j}\right]\right|^{2},
$$

the sum of covariances above over indices $|j| \leq 2 b$ is bounded by $C m^{-2 / 3}\left(b-b_{1}\right)$ (i.e., $\left|\operatorname{Cov}\left[\bar{Y}_{1, b_{1}}, \bar{Y}_{1+j, b_{1}}\right]\right| \leq C b^{-1}$ and $\left.\left|\operatorname{Cov}\left[\bar{Y}_{1, b_{1}}, b^{-1} S_{1+j}\right]\right| \leq C\left(b-b_{1}\right) / b\right)$ while the sum over $|j|>2 b$ is bounded by $m^{-2 / 3} \sum_{k=b}^{\infty}(k / b) \alpha(k)^{\delta /(2 r+\delta)} \leq C m^{-1}$ by Lemma 1(ii) and condition $M_{r}$; hence,

$$
\begin{aligned}
& \left|C_{b, b_{1}, m}-\frac{2\left(b_{1}\right)^{3}}{b\left(m-b_{1}+1\right)^{2}} \sum_{i, j=1}^{m-b_{1}+1}\left[\operatorname{Cov}\left(\bar{Y}_{i, b_{1}}, \bar{Y}_{j, b_{1}}\right)\right]\right| \\
\leq & C \frac{b b_{1}}{m} D_{b, b_{1}, m} \leq C\left[m^{-1}\left(b-b_{1}\right)+m^{-4 / 3}\right] .
\end{aligned}
$$

Also, from the proof of Theorem 5(ii),

$$
\left|\frac{2 b_{1}^{2}}{\left(m-b_{1}+1\right)^{2}} \sum_{i, j=1}^{m-b_{1}+1}\left[\operatorname{Cov}\left(\bar{Y}_{i, b_{1}}, \bar{Y}_{j, b_{1}}\right)\right]^{2}-V_{0} \frac{b_{1}}{m}\right| \leq C m^{-4 / 3} .
$$

Now by (A.15) and using (A.17) through (A.20), we have (since $b-b_{1} \geq 1$ )

$$
\begin{aligned}
c_{b, b_{1}, m}(0) & \leq C \frac{b-b_{1}}{m^{1 / 3}}+2 V_{0}\left|\frac{b^{2}}{m^{2 / 3}}-\frac{b_{1}^{2}}{m^{2 / 3}}\right|+4 V_{0}^{2}\left|\frac{b_{1}^{4}}{b^{2} m^{2 / 3}}-\frac{b_{1}^{2}}{m^{2 / 3}}\right| \\
& \leq C \frac{b-b_{1}}{m^{1 / 3}}
\end{aligned}
$$

as $\left|b^{2}-b_{1}^{2}\right| \leq C m^{1 / 3}\left|b-b_{1}\right|$. This proves (A.16) and consequently (A.14).

Since

$$
\begin{aligned}
& \operatorname{Var}\left\{\left[\left(\frac{n}{m}\right)^{1 / 2} m^{2 / 3}\left[\Delta_{m}^{(1), \infty}(b)-\Delta_{m}^{(1), \infty}\left(b_{1}\right)\right]\right]^{2}\right\} \\
= & 2\left[m^{4 / 3} \frac{n}{m} \operatorname{Var}\left(\Delta_{m}^{(1), \infty}(b)-\Delta_{m}^{(1), \infty}\left(b_{1}\right)\right)\right]^{2}+c_{b, b_{1}, n}^{(4)},
\end{aligned}
$$

where $c_{b, b_{1}, n}^{(4)}$ denotes the fourth-order cumulant of $(n / m)^{1 / 2} m^{2 / 3}\left[\Delta_{m}^{(1), \infty}(b)-\Delta_{m}^{(1), \infty}\left(b_{1}\right)\right]$, then by (A.14) it suffices to show $\left|c_{b, b_{1}, n}^{(4)}\right| \leq C\left(b-b_{1}\right)^{2} m^{-2 / 3}, b, b_{1} \in \mathcal{J}_{m}$, to complete 
the proof of Lemma 3(ii). Using Lemma 1(i-ii), (A.12), and condition $M_{r}\left(r=14+4 a_{0}\right)$, it can be verified that $\left|c_{b, b_{1}, n}^{(4)}\right| \leq C m / n \leq C\left(b-b_{1}\right)^{2} m^{-2 / 3}$, with the second inequality from $m^{5 / 3} / n=O(1)$.

To establish Lemma 3(iv), where $\mathrm{E}\left[\Omega_{1, m}(b)\right]^{2}=\operatorname{Var}\left[\Omega_{1, m}(b)\right] \leq \mathrm{E}\left[\Omega_{3, m}(b)\right]^{2}$, it can first be shown that, for $b \in \mathcal{J}_{m}$,

$$
\mathrm{E}\left[W_{m}^{4}(b)\right] \leq C, \quad W_{m}(b) \equiv \frac{(n / m)^{1 / 2} m^{2 / 3}}{n-m+1} \sum_{i=1}^{n-m+1}\left[\hat{\sigma}_{i, m}^{2}(b)-\mathrm{E}\left[\hat{\sigma}_{i, m}(b)\right]\right],
$$

following from $\left|\mathrm{cu}\left[W_{m}(b), W_{m}(b), W_{m}(b), W_{m}(b)\right]\right| \leq C$ and $\mathrm{E}\left[W_{m}^{2}(b)\right]=\operatorname{Var}\left[W_{m}(b)\right] \leq C$ by $\mathrm{E} W_{m}(b)=0$, (A.11)-(A.12), Lemma 1(i-ii) and condition $M_{r}$. By similar mixing and moment bounds, $\mathrm{E}\left[\hat{\sigma}_{n}^{2}(\tilde{\ell})-\sigma_{\infty}^{2}\right]^{4} \leq C\left[\tilde{\ell}_{n}^{-4}+\left(\tilde{\ell}_{n} / n\right)^{2}\right]$ analogously to (A.12). Using this moment bound with the one on $\mathrm{E}\left[W_{m}^{4}(b)\right]$, the Cauchy-Schwarz inequality yields $\mathrm{E}\left\{\left[(n / m)^{1 / 2} m^{2 / 3} \Omega_{3, m}(b)\right]^{2}\right\} \leq C\left[\tilde{\ell}_{n}^{-2}+\left(\tilde{\ell}_{n} / n\right)\right] \leq C m^{-1}$, by the block growth assumptions.

Lemma 3(v) follows from $\left|\Omega_{2, m}(b)-\Omega_{2, m}\left(b_{1}\right)\right| \leq 2\left|\hat{\sigma}_{n}^{2}\left(\tilde{\ell}_{n}\right)-\mathrm{E}\left[\hat{\sigma}_{n}^{2}\left(\tilde{\ell}_{n}\right)\right]\right| m^{-2 / 3}\left|b-b_{1}\right|$, using Theorem $5(\mathrm{i})$ to bound $\left|\mathrm{E}\left[\hat{\sigma}_{1, m}^{2}(b)\right]-\mathrm{E}\left[\hat{\sigma}_{1, m}^{2}\left(b_{1}\right)\right]\right|$ and the fact that $\mathrm{E} \mid \hat{\sigma}_{n}^{2}\left(\tilde{\ell}_{n}\right)-$ $\left.\mathrm{E}\left[\hat{\sigma}_{n}^{2}\left(\tilde{\ell}_{n}\right)\right]\right|^{2} \leq C \tilde{\ell}_{n} / n$ by Theorem $5($ ii).

\section{A.3. A further auxiliary theorem (Theorem 6)}

Theorem 6 next establishes the general consistency of the HHJ block estimator (and its oracle-version), at both sample and subsample levels, and also provides some tightness results for developing probabilistic bounds on the HHJ block estimator (cf. Theorem 2).

Recall $\Delta_{m}^{\infty}(b), b \in \mathcal{J}_{m}$, from (A.8) and that $\Delta_{m}(b)$ is defined by replacing $\widehat{\operatorname{MSE}}_{m}^{\infty}(\cdot)$ with $\widehat{\operatorname{MSE}}_{m}(\cdot)$ in (A.8) so that $\Delta_{m}(b)=\Delta_{m}^{\infty}(b)+\Omega_{1, m}(b)+\Omega_{2, m}(b)$ holds for $b \in \mathcal{J}_{m}$ from (A.10). Given any $C>0$, define a block set $\mathcal{J}_{m}^{\text {opt }}(C)=\left\{b \in \mathcal{J}_{m}:\left|b_{m}^{\text {opt }}-b\right| \leq C m^{1 / 3}\right\}$.

Theorem 6. Suppose that conditions $D, M_{r}$ with $r=14+4 a_{0}$, and $S$ hold, with $a_{0}$ as specified by condition D. Assume that $m^{-1}+m / n \rightarrow 0$ with $m^{5 / 3} / n=O(1)$ as $n \rightarrow \infty$ and that $\tilde{\ell}_{n}$ in the HJJ method satisfies $\tilde{\ell}_{n}^{-1}+\tilde{\ell}_{n}^{2} / n \rightarrow 0$ and $m\left(\tilde{\ell}_{n}^{-2}+n^{-1} \tilde{\ell}_{n}\right)=O(1)$. Let $\Lambda_{m}(b), b \in \mathcal{J}_{m}$ denote either $\Delta_{m}^{\infty}(b)$ or $\Delta_{m}^{\infty}(b)+\Omega_{1, m}(b)$. Then,

(i) there exists an integer $N_{0} \geq 1$ and constant $A>0$ such that

$$
\begin{aligned}
P\left(a_{n}^{-1 / 2} m^{2 / 3}\left(\frac{n}{m}\right)^{1 / 2} \max _{b \in \mathcal{J}_{m}^{\text {opt }}\left(a_{n}\right)}\left|\Lambda_{m}(b)\right|>\lambda\right) & \leq \frac{A}{\lambda}, \\
P\left(a_{n}^{-1} m^{2 / 3}\left(\frac{m^{1 / 3}}{\tilde{\ell}_{n}} \frac{n}{m}\right)^{1 / 2} \max _{b \in \mathcal{J}_{m}^{\text {opt }}\left(a_{n}\right)}\left|\Omega_{2, m}(b)\right|>\lambda\right) & \leq \frac{A}{\lambda},
\end{aligned}
$$

holds for any $\lambda>0$, any $n \geq N_{0}$ and any positive $a_{n}>0$.

(ii) $\hat{b}_{m, \mathrm{HHJ}}^{\mathrm{opt}} / b_{m}^{\mathrm{opt}} \stackrel{p}{\rightarrow} 1$ and $\hat{b}_{m, \mathrm{HHJ}}^{\mathrm{opt}, \infty} / b_{m}^{\mathrm{opt}} \stackrel{p}{\rightarrow} 1$ as $n \rightarrow \infty$.

(iii) $\hat{\ell}_{n, \mathrm{HHJ}}^{\mathrm{opt}} / \ell_{n}^{\mathrm{opt}} \stackrel{p}{\rightarrow} 1$ and $\hat{\ell}_{n, \mathrm{HHJ}}^{\mathrm{opt}, \infty} / \ell_{n}^{\mathrm{opt}} \stackrel{p}{\rightarrow} 1$ as $n \rightarrow \infty$

imsart-bj ver. 2011/12/06 file: BEJ511.supplementary.material.tex date: February 8, 2013 
Proof of Theorem 6. We shall establish Theorem 6(ii)-(iii) firstly, which is not involved in showing Theorem $6(\mathrm{i})$. Set $b_{m}^{0}=\mathcal{C}_{0} m^{1 / 3}$ as the minimizer of $f_{m}(y), y>0$ (i.e., $b_{m}^{0}$ subsample version of $\ell_{n}^{0}=\mathcal{C}_{0} n^{1 / 3}, \mathcal{C}_{0}=\left[2 B_{0}^{2} / V_{0}\right]^{1 / 3}$, from (2.3) solving $\left.d\left[f_{m}\left(b_{m}^{0}\right)\right] / d y=0\right)$. We shall consider $\hat{b}_{m, \mathrm{HHJ}}^{\mathrm{opt}}$; the proof for $\hat{b}_{m, \mathrm{HHJ}}^{\mathrm{opt}, \infty}$ is similar. Using Lemma 2 and the definition of $\Delta_{m}(b)$, it holds that $0 \leq m^{2 / 3}\left[\operatorname{MSE}_{m}\left(\hat{b}_{m, \mathrm{HHJ}}^{\mathrm{opt}}\right)-\operatorname{MSE}_{m}\left(b_{m}^{\text {opt }}\right)\right] \leq C m^{-2 / 3}+$ $C m^{2 / 3} \max _{b \in \mathcal{J}_{m}}\left|\Delta_{m}(b)\right|=o_{p}(1)$, where the convergence in probability follows from Theorem 6(i) [e.g., $\max _{b \in \mathcal{J}_{m}}\left|\Delta_{m}(b)\right|=O_{p}\left((m / n)^{1 / 2}+\left(\tilde{\ell}_{n} m^{2 / 3} / n\right)^{1 / 2}\right)=o_{p}(1)$ ] and the block growth assumptions. From Theorem $1(\mathrm{i}-\mathrm{ii})$, it then holds that $0 \leq m^{2 / 3}\left[f_{m}\left(\hat{b}_{m, \mathrm{HHJ}}^{\mathrm{opt}}\right)-\right.$ $\left.f_{m}\left(b_{m}^{0}\right)\right]=o_{p}(1)$, from which $\hat{b}_{m, \mathrm{HHJ}}^{\text {opt }} / b_{m}^{0} \stackrel{p}{\rightarrow} 1$ follows (e.g., any subsequence of $\hat{b}_{m}^{\text {opt }} / b_{m}^{0}$ has a further subsequence converging almost surely). It then holds immediately that $\hat{\ell}_{n, \mathrm{HHJ}}^{\mathrm{opt}} / \ell_{n}^{0} \stackrel{p}{\rightarrow} 1$ from $(2.7)$ and $\ell_{n}^{0}=b_{m}^{0}(n / m)^{1 / 3}$ in $(2.3)$. Both $b_{m}^{\text {opt }} / b_{m}^{0} \rightarrow 1$ and $\ell_{n}^{\text {opt }} / \ell_{n}^{0} \rightarrow$ 1 hold by Theorem 1(ii), establishing Theorem 6(ii-iii) for the HHJ block estimators.

To prove Theorem 6(i), fix $\lambda>1, a_{n}>0$ and large $n \geq N_{0}$ under Lemma 3. Consider $\Lambda_{m}(b)=\Delta_{m}^{\infty}(b)+\Omega_{1, m}(b), b \in \mathcal{J}_{m}$ (the case with $\Lambda_{m}(b)=\Delta_{m}^{\infty}(b)$ being nearly the same). Then, recalling (A.9), we have

$$
P\left(m^{2 / 3}\left(\frac{n}{m}\right)^{1 / 2} \max _{b \in \mathcal{J}_{m}^{\text {opt }}\left(a_{n}\right)}\left|\Lambda_{m}(b)\right|>a_{n}^{1 / 2} \lambda\right) \leq \sum_{i=1}^{3} p_{i, n, \lambda}
$$

where $p_{i, n, \lambda} \equiv P\left[m^{2 / 3}(n / m)^{1 / 2} \max _{b \in \mathcal{J}_{m}^{\text {opt }}\left(a_{n}\right)}\left|\Delta_{m}^{(i), \infty}(b)\right|>a_{n}^{1 / 2} \lambda / 3\right], i=1,2$ and $p_{3, n, \lambda} \equiv$ $P\left[m^{2 / 3}(n / m)^{1 / 2} \max _{b \in \mathcal{J}_{m}^{\text {opt }}\left(a_{n}\right)}\left|\Omega_{1, m}(b)\right|>a_{n}^{1 / 2} \lambda / 3\right]$. Assume $a_{n} \geq m^{-1 / 3} ;$ if $a_{n}<m^{-1 / 3}$, then each $p_{i, n, \lambda}=0$ since $\mathcal{J}_{m}^{\text {opt }}\left(a_{n}\right)=\left\{b_{m}^{\text {opt }}\right\}$ with $\Lambda_{m}\left(b_{m}^{\text {opt }}\right)=0$ by definition. To prove the first statement in Theorem 6(i), it suffices to show that each $p_{i, n, \lambda} \leq C / \lambda$ for $C$ independent of $m, n$.

By Lemma 3(ii) and (iv),

$$
\begin{aligned}
& p_{2, n, \lambda} \leq \frac{9}{a_{n} \lambda^{2}} \frac{n}{m} m^{4 / 3} \sum_{b \in \mathcal{J}_{m}^{\text {opt }}\left(a_{n}\right)} \mathrm{E}\left\{\left[\Delta_{m}^{(2), \infty}(b)\right]^{2}\right\} \leq \frac{C}{\lambda^{2}} \\
& p_{3, n, \lambda} \leq \frac{9}{a_{n} \lambda^{2}} \frac{n}{m} m^{4 / 3} \sum_{b \in \mathcal{J}_{m}^{\text {opt }}\left(a_{n}\right)} \mathrm{E}\left\{\left[\Omega_{1, m}(b)\right]^{2}\right\} \leq \frac{C}{\lambda^{2}} .
\end{aligned}
$$

Next bound $p_{1, n, \lambda} \leq p_{1, n, \lambda}^{+}+p_{1, n, \lambda}^{-}$, where

$$
p_{1, n, \lambda}^{+} \equiv P\left(m^{2 / 3}(n / m)^{1 / 2} \max _{b \in \mathcal{J}_{m}^{\text {opt }}\left(a_{n}\right), b \geq b_{m}^{\text {opt }}}\left|\Delta_{m}^{(1), \infty}(b)\right|>a_{n}^{1 / 2} \lambda / 6\right)
$$

and $p_{1, n, \lambda}^{-}$is analogously defined for $b \in \mathcal{J}_{m}^{\text {opt }}\left(a_{n}\right), b \leq b_{m}^{\text {opt }}$. Let $k_{n}=\left\lfloor a_{n} m^{1 / 3}\right\rfloor \geq 1$ and, for $j=0, \ldots, k_{n}$, define $S_{j} \equiv \sum_{i=0}^{j} \xi_{i}=\Delta_{m}^{(1), \infty}\left(b_{m}^{\text {opt }}+j\right)$, where $\xi_{0}=0$ and $\xi_{i} \equiv m^{2 / 3}(n / m)^{1 / 2}\left[\Delta_{m}^{(1), \infty}\left(b_{m}^{\mathrm{opt}}+i\right)-\Delta_{m}^{(1), \infty}\left(b_{m}^{\mathrm{opt}}+i-1\right)\right], i=1, \ldots, k_{n}$. Then, define 
$M_{k_{n}} \equiv \max _{0 \leq j \leq k_{n}}\left|S_{j}\right|$ so that, by combining Lemma 3(iii) and Lemma 1(iii) (with $\gamma=4$, $\beta=2$ there),

$$
p_{1, n, \lambda}^{+}=P\left(M_{k_{n}}>a_{n}^{1 / 2} \lambda / 6\right) \leq \frac{C}{a_{n}^{2} \lambda^{4}} \frac{k_{n}^{2}}{m^{2 / 3}} \leq \frac{C}{\lambda^{4}} .
$$

Note that $p_{1, n, \lambda}^{-}$can be similarly bounded, re-defining $M_{k_{n}}$ with sums $S_{k}$ using $\xi_{i, m} \equiv$ $m^{2 / 3}(n / m)^{1 / 2}\left[\Delta_{m}^{(1), \infty}\left(b_{m}^{\mathrm{opt}}-j\right)-\Delta_{m}^{(1), \infty}\left(b_{m}^{\mathrm{opt}}-j+1\right)\right], j=1, \ldots, k_{n}$. This establishes the first part of Theorem 6(i).

For the second statement in Theorem 6(i), we may likewise consider splitting and bounding $(n / m)^{1 / 2} m^{2 / 3} \max _{b \in \mathcal{J}_{m}^{\text {opt }}\left(a_{n}\right)}\left|\Omega_{2, m}(b)\right|$ over the cases $b \in \mathcal{J}_{m}^{\text {opt }}\left(a_{n}\right)$ with $b \geq b_{m}^{\text {opt }}$ or $b \leq b_{m}^{\text {opt }}$. Re-defining $M_{k_{n}}$ with sums $S_{j}$ for $\xi_{i} \equiv m^{2 / 3}(m / n)^{1 / 2}\left[\Omega_{2, m}\left(b_{m}^{\text {opt }}+i\right)-\right.$ $\left.\Omega_{2, m}\left(b_{m}^{\text {opt }}+i-1\right)\right], i=1, \ldots, k_{n}$, we have

$$
\begin{aligned}
& P\left[\left(\frac{n}{m}\right)^{1 / 2} m^{2 / 3} \max _{b \in \mathcal{J}_{m}^{\text {opt }}\left(a_{n}\right), b \geq b_{0}}\left|\Omega_{2, m}(b)\right|>\lambda a_{n}\left(\frac{\tilde{\ell}_{n}}{m^{1 / 3}}\right)^{1 / 2}\right] \\
= & P\left[M_{k_{n}}>\lambda a_{n}\left(\frac{\tilde{\ell}_{n}}{m^{1 / 3}}\right)^{1 / 2}\right] \leq \frac{C m^{1 / 3}}{\tilde{\ell}_{n} a_{n} \lambda^{2}} \frac{\tilde{\ell}_{n} k_{n}^{2}}{m} \leq \frac{C}{\lambda^{2}},
\end{aligned}
$$

by Lemma 3 (v) and Lemma 1 (iii) (with $\gamma=2, \beta=2$ there). The case with $b \leq b_{m}^{\text {opt }}$ is similarly handled.

\section{Appendix B: Proofs of the HHJ/NPPI block estimator convergence results}

Here we provide proofs of Theorem 2(ii) and Theorem 3, with the proof of Theorem 2(i) appearing in Section 7 of the main manuscript.

Proof of Theorem 2(ii) (HHJ method). The proof follows that of Theorem 2(i) (Section 7) with modifications. As the analog to (7.6) and (7.7), we now have

$$
0 \leq m^{2 / 3}\left[f_{m}\left(\hat{b}_{m, \mathrm{HHJ}}^{\mathrm{opt}}\right)-f_{m}\left(b_{m}^{0}\right)\right] \leq C m^{-2 / 3}+m^{2 / 3}\left[\operatorname{MSE}_{m}\left(\hat{b}_{m, \mathrm{HHJ}}^{\mathrm{opt}}\right)-\operatorname{MSE}\left(b_{m}^{\mathrm{opt}}\right)\right]
$$

and, using $\Delta_{m}(\cdot)$ from (A.10) with Lemma 2 with the conditions on $m, n, \tilde{\ell}_{n}$,

$$
\begin{aligned}
0 & \leq m^{2 / 3}\left[\operatorname{MSE}_{m}\left(\hat{b}_{m, \mathrm{HHJ}}^{\mathrm{opt}}\right)-\operatorname{MSE}_{m}\left(b_{m}^{\mathrm{opt}}\right)\right] \\
& \leq m^{2 / 3} \Delta_{m}\left(\hat{b}_{m, \mathrm{HHJ}}^{\mathrm{opt}}\right)+\frac{m^{2 / 3}}{\tilde{\ell}_{n}}\left|B_{0} \frac{b_{m}^{\mathrm{opt}}-\hat{b}_{m, \mathrm{HHJ}}^{\mathrm{opt}}}{b_{m}^{\mathrm{opt}} \hat{b}_{m, \mathrm{HHJ}}^{\mathrm{opt}}}+\sigma_{\infty}^{2} \frac{b_{m}^{\mathrm{opt}}-\hat{b}_{m, \mathrm{HHJ}}^{\mathrm{opt}}}{m}\right|+\frac{C}{m^{2 / 3}}
\end{aligned}
$$

imsart-bj ver. 2011/12/06 file: BEJ511.supplementary.material.tex date: February 8, 2013 
holds for some $C>0$ (note $\Delta_{m}\left(\hat{b}_{m, \mathrm{HHJ}}^{\mathrm{opt}}\right) \geq 0$ by definition). As a counterpart to (7.9), when $\left|\hat{b}_{m, \mathrm{HHJ}}^{\mathrm{opt}} / b_{m}^{0}-1\right|<1 / 2$, then

$$
\left(\frac{\hat{b}_{m, \mathrm{HHJ}}^{\mathrm{opt}}-b_{m}^{\mathrm{opt}}}{m^{1 / 3}}\right)^{2} \leq 2^{-1} C_{0} \max \left\{m^{-2 / 3}, m^{2 / 3} / \tilde{\ell}_{n}^{2}, m^{2 / 3} \Delta_{m}\left(\hat{b}_{m, \mathrm{HHJ}}^{\mathrm{opt}, \infty}\right)\right\},
$$

holds for some large $C_{0}>1$ (independent of $n, m, b \in \mathcal{J}_{m}$ ), noting that $b^{-1} m^{1 / 3}$ is uniformly bounded in $b \in \mathcal{J}_{m}$. Hence, for all $C_{1} \geq C_{0}$ and setting $m_{\ell} \equiv \max \left\{m^{-2 / 3}, m^{2 / 3} / \tilde{\ell}_{n}^{2}\right\}$, it follows that

$$
\begin{aligned}
& \left(\frac{\hat{b}_{m, \mathrm{HHJ}}^{\mathrm{opt}}-b_{m}^{\mathrm{opt}}}{m^{1 / 3}}\right)^{2} \\
\leq & C_{1} \max \left\{m_{\ell}, m^{2 / 3}\left|\Lambda_{m}\left(\hat{b}_{m, \mathrm{HHJ}}^{\mathrm{opt}}\right)\right|, m^{2 / 3}\left|\Omega_{2, m}\left(\hat{b}_{m, \mathrm{HHJ}}^{\mathrm{opt}}\right)\right|\right\}
\end{aligned}
$$

when $\left|\hat{b}_{m, \mathrm{HHJ}}^{\mathrm{opt}} / b_{m}^{0}-1\right|<1 / 2$ (holding in probability by Theorem $6(\mathrm{ii})$ ), using $\Delta_{m}(b)=$ $\Lambda_{m}(b)+\Omega_{2, m}(b), \Lambda_{m}(b) \equiv \Delta_{m}^{\infty}(b)+\Omega_{1, m}(b), b \in \mathcal{J}_{m}$ from (A.10). Assume (B.21) to hold along with $\mathcal{J}_{m}^{\text {opt }}\left(C_{0}\right)=\mathcal{J}_{m}$. As in the proof of Theorem 1(i), we construct recursive events to cage $\hat{b}_{m, \mathrm{HHJ}}^{\text {opt }}$ in potentially finer neighborhoods around $b_{m}^{\text {opt }}$ with high probability.

Fix $C_{1}>C_{0}$, set $a_{0, n}=C_{1}^{2}$ and $L \equiv\lceil 2 \log \log n\rceil$, and define

$$
a_{i, n}^{2} \equiv C_{1}^{4} \max \left\{m_{\ell}, 2^{\sum_{k=0}^{i-1}[(L-i+1)+k] 2^{-k}}\left(\frac{\tilde{\ell}_{n}}{m^{1 / 3}} \frac{m}{n}\right)^{2^{-1} \sum_{k=0}^{i-1} 2^{-k}}\right\}, \quad i \geq 1,
$$

assuming $\log \log n>1$. Define an integer $J$ such that

$$
J=\min \left\{i=1, \ldots, L+1: a_{i, n}^{2}=C_{1}^{4} m_{\ell}\right\} .
$$

and let $J=L+1$ if the above integer set is empty. For $i=0, \ldots, J-1$, let $A_{i}$ be the event

$$
\begin{aligned}
& \max _{b \in \mathcal{J}_{m}^{\text {opt }}\left(a_{i, n}\right)} m^{2 / 3}\left|\Lambda_{m}(b)\right| \leq a_{i, n}^{1 / 2}(m / n)^{1 / 2} \lambda_{i} \quad \& \\
& \max _{b \in \mathcal{J}_{m}^{\text {opt }}\left(a_{i, n}\right)} m^{2 / 3}\left|\Omega_{2, m}(b)\right| \leq a_{i, n}\left(\tilde{\ell}_{n} / m^{1 / 3}\right)^{1 / 2}(m / n)^{1 / 2} \lambda_{i}, \quad \lambda_{i} \equiv C_{1}^{1 / 2} 2^{L-i}
\end{aligned}
$$

and let $B_{i}, i \geq 1$, be the event

$$
\left(\frac{\hat{b}_{m, \mathrm{HHJ}}^{\mathrm{opt}}-b_{m}^{\mathrm{opt}}}{m^{1 / 3}}\right)^{2} \leq a_{i, n}^{2}
$$

Since $\mathcal{J}_{m}^{\text {opt }}\left(a_{0, n}\right)=\mathcal{J}_{m}$, event $A_{0}$ implies $B_{1}$ by (B.21); also, for $J>1$, if $A_{i} \cap B_{i}$ holds, then so must $B_{i+1}$ for $i=1, \ldots, J-1$, which follows by (B.21) and the fact that 
$\left(m^{1 / 3} / \tilde{\ell}_{n}\right)^{1 / 2} \leq a_{i, n}^{1 / 2}$ and consequently $a_{i, n}^{1 / 2}(m / n)^{1 / 2} \leq a_{i, n}\left(\tilde{\ell}_{n} / m^{1 / 3}\right)^{1 / 2}(m / n)^{1 / 2}$ hold for $i=1, \ldots, J-1$ by construction.

Suppose now that $A_{J} \cap B_{J}$ holds for an event $A_{J}$ defined as

$$
\begin{aligned}
\max _{b \in \mathcal{J}_{m}^{\text {opt }}\left(a_{J, n}\right)} m^{2 / 3}\left|\Lambda_{m}(b)\right| \leq a_{n, J}^{1 / 2}(m / n)^{1 / 2} C_{1}^{1 / 2} \& \\
\max _{b \in \mathcal{J}_{m}^{\text {opt }}\left(a_{J, n}\right)} m^{2 / 3}\left|\Lambda_{m}(b)\right| \leq a_{n, J}\left(\tilde{\ell}_{n} / m^{1 / 3}\right)^{1 / 2}(m / n)^{1 / 2} C_{1}^{1 / 2} ;
\end{aligned}
$$

the complement $\left(A_{J} \cap B_{J}\right)^{c}$ has probability bounded by

$$
\sum_{i=0}^{J} P\left(A_{i}^{c}\right) \leq 2 A C_{1}^{-1 / 2}\left(1+\sum_{k=0}^{L} 2^{-k}\right) \leq 6 A C_{1}^{-1 / 2}
$$

by Theorem 6(i)-(ii), and so can be made arbitrarily small for large $C_{1}$. When $A_{J} \cap B_{J}$ holds, then by construction (B.21) further implies that either

$$
\begin{aligned}
& \left(\frac{\hat{b}_{m, \mathrm{HHJ}}^{\mathrm{opt}}-b_{m}^{\mathrm{opt}}}{m^{1 / 3}}\right)^{2} \\
\leq & C_{1}^{7 / 2} \max \left\{m_{\ell}, m_{\ell}^{1 / 4}(m / n)^{1 / 2}, m_{\ell}^{1 / 2}\left(\tilde{\ell}_{n} / m^{1 / 3}\right)^{1 / 2}(m / n)^{1 / 2}\right\} \\
\leq & C_{1}^{4} \max \left\{m_{\ell},\left(\tilde{\ell}_{n} / n\right)^{1 / 2}, m^{1 / 3} / n^{1 / 2}, m^{2 / 3} /\left(\tilde{\ell}_{n} n\right)^{1 / 2}\right\}
\end{aligned}
$$

if $a_{J, n}^{2}=C_{1}^{4} m_{\ell}$, or the remaining possibility is $a_{J, n} \neq C_{1}^{2} m_{\ell}$ in event $A_{J}, J=L+1$ and $1 \leq a_{J, n}^{1 / 2}\left(\tilde{\ell}_{n} / m^{1 / 3}\right)^{1 / 2}$ so that

$$
\begin{aligned}
\left(\frac{\hat{b}_{m, \mathrm{HHJ}}^{\mathrm{opt}}-b_{m}^{\mathrm{opt}}}{m^{1 / 3}}\right)^{2} & \leq C_{1}^{7 / 2} \max \left\{m_{\ell}, 2^{\sum_{k=0}^{L} k 2^{-k}}\left(\frac{\tilde{\ell}}{m^{1 / 3}} \frac{m}{n}\right)^{2^{-1} \sum_{k=0}^{L} 2^{-k}}\right\} \\
& \leq 4 C_{1}^{4} \max \left\{m_{\ell}, \frac{\tilde{\ell}}{m^{1 / 3}} \frac{m}{n}\right\}
\end{aligned}
$$

using that $\left(\tilde{\ell}_{n} m^{2 / 3} / n\right)^{2^{-1}} \sum_{k=0}^{L} 2^{-k}-1 \leq 1$ and $2^{\sum_{k=1}^{L} k 2^{-k}} \leq 4$ for $L=2\lceil\log \log n\rceil$. We now have shown

$$
\left|\frac{\hat{b}_{m, \mathrm{HHJ}}^{\mathrm{opt}}-b_{m}^{\mathrm{opt}}}{m^{1 / 3}}\right|=O_{p}\left(\max \left\{m^{-1 / 3}, \frac{m^{1 / 3}}{\tilde{\ell}_{n}}, \frac{m^{1 / 6}}{n^{1 / 4}}, \frac{m^{1 / 3}}{\left(\tilde{\ell}_{n} n\right)^{1 / 4}}, \frac{\tilde{\ell}_{n}^{1 / 4}}{n^{1 / 4}}, \frac{m^{1 / 3} \tilde{\ell}_{n}^{1 / 2}}{n^{1 / 2}}\right\}\right)
$$

from which Theorem 2(ii) now follows (as in the proof of Theorem 2(i)).

Proof of Theorem 3 (NPPI method). As the NPPI method involves variance $\hat{V}_{0}$ and bias $\hat{B}_{0}$ quantity estimators (i.e, plugged into the large-sample optimal block formula

imsart-bj ver. 2011/12/06 file: BEJ511.supplementary.material.tex date: February 8, 2013 
$\ell_{n}^{0}=\left[2 B_{0}^{2} / V_{0}\right]^{1 / 3} n^{1 / 3}$ from $\left.(2.3)\right)$, we need only consider $\hat{V}_{0}$ here as $\hat{B}_{0}$ is treated in Section 7 of the main manuscript.

For notational simplicity in considering $\hat{V}_{0}$, we shall set $\ell_{1}=\ell, \hat{\sigma}_{n}^{2}(\ell)=\hat{\varphi}_{n}$ and define the JAB variance estimator (e.g., the Jackknife point values) in terms of $\hat{\varphi}_{n}$; see Section 2.3. Also, set $\hat{\varphi}_{n}^{(0)}=\hat{\varphi}_{n}$ and $p=n / \ell$. Recall that $N=n-\ell+1$ and $M=N-m+1$. Then, for $i=0,1, \ldots, M$, using a third order Taylor's expansion of $H\left(\bar{X}_{n, i}^{*}\right)$ around $\mu(=0)$, we can write

$$
\hat{\varphi}_{n}^{(i)}=\mathrm{E}_{*}\left(\nabla^{\prime} U_{1, i}^{*}\right)^{2}+\hat{R}_{1 n}^{(i)}
$$

where $U_{1, i}^{*}=\left(X_{1}^{* i}+\ldots+X_{\ell}^{* i}\right) / \sqrt{\ell}$ (a scaled sum of a resampled block from the $i$ th reduced block collection) and $\hat{R}_{1 n}^{(i)}$ is a remainder term satisfying

$$
\begin{aligned}
& \left|\hat{R}_{1 n}^{(i)}-n\left[-\frac{1}{p}\left(\nabla^{\prime} \hat{\mu}_{n, i}\right)^{2}+\operatorname{Cov}_{*}\left(\nabla^{\prime} \bar{X}_{n, i}^{*}, \sum_{\|\beta\|_{1}=2} c_{\beta}\left(\bar{X}_{n, i}^{*}\right)^{\beta}\right)\right]\right| \\
\leq & C n\left\{\mathrm{E}_{*}\left\|\bar{X}_{n, i}^{*}\right\|^{4}+\mathrm{E}_{*}\left\|\bar{X}_{n, i}^{*}\right\|^{6+2 a_{0}}\right\}
\end{aligned}
$$

for $\hat{\mu}_{n, i}=\mathrm{E}_{*} \bar{X}_{n, i}^{*}$. Hence, for $1 \leq i \leq M$,

$$
\tilde{\varphi}_{n}^{(i)}=m^{-1}\left(N \hat{\varphi}_{n}-(N-m) \hat{\varphi}_{n}^{(i)}\right)=m^{-1} \sum_{j=i}^{j+m-1} W_{j}+\tilde{R}_{1 n}^{(i)}
$$

where $\tilde{R}_{1 n}^{(i)}=m^{-1}\left[N \hat{R}_{1 n}^{(0)}-(N-m) \hat{R}_{1 n}^{(i)}\right]$ and $W_{j}=\left(\nabla^{\prime} U_{1, j}\right)^{2}$, with $U_{1, j}=\left(X_{j}+\ldots+\right.$ $\left.X_{j+\ell-1}\right) / \sqrt{\ell}$. We next write $\bar{W}_{m, i}=m^{-1} \sum_{j=i}^{i+m-1}\left(W_{j}-\mathrm{E} W_{j}\right), 1 \leq j \leq M$. Then, it follows that

$$
\begin{aligned}
& \left|\widehat{\operatorname{VAR}}-\operatorname{Var}\left(\hat{\varphi}_{n}\right)\right| \\
= & \left|\frac{m}{N-m} \frac{1}{M} \sum_{i=1}^{M}\left(\tilde{\varphi}_{n}^{(i)}-\hat{\varphi}_{n}\right)^{2}-\operatorname{Var}\left(\hat{\varphi}_{n}\right)\right| \\
= & \left|\frac{m}{N-m} \frac{1}{M} \sum_{i=1}^{M}\left\{\bar{W}_{m, i}+\tilde{R}_{1 n}^{(i)}-\left(\hat{\varphi}_{n}-\mathrm{E} W_{1}\right)\right\}^{2}-\operatorname{Var}\left(\hat{\varphi}_{n}\right)\right| \\
\leq & \frac{m}{N-m}\left|\frac{1}{M} \sum_{i=1}^{M}\left[\bar{W}_{m, i}^{2}-\mathrm{E} \bar{W}_{m, i}^{2}\right]\right|+\frac{m}{N-m} \frac{1}{M} \sum_{i=1}^{M}\left|\tilde{R}_{1 n}^{(i)}\right|^{2} \\
& +\frac{m}{N-m}\left|\hat{\varphi}_{n}-\mathrm{E} W_{1}\right|^{2}+\frac{2 m}{N-m}\left|\frac{1}{M} \sum_{i=1}^{M} \bar{W}_{m, i} \tilde{R}_{1 n}^{(i)}\right| \\
& +\frac{2 m}{N-m}\left|\hat{\varphi}_{n}-\mathrm{E} W_{1}\right|\left|\frac{1}{M} \sum_{i=1}^{M} \bar{W}_{m, i}\right|+\left|\operatorname{Var}\left(\hat{\varphi}_{n}\right)-\frac{m}{N-m} \mathrm{E} \bar{W}_{m, 1}^{2}\right| \\
\equiv & I_{1}+I_{2}+\ldots+I_{6} \quad \text { (say). }
\end{aligned}
$$

imsart-bj ver. 2011/12/06 file: BEJ511.supplementary.material.tex date: February 8, 2013 
Of these 6 terms, we only present the details of the derivations of upper bounds on $I_{1}, I_{3}$ and $I_{6}$, which are the terms of leading orders. The other terms, $I_{2}, I_{4}$ and $I_{5}$, are negligible; we only give an outline of the proof for these to save space.

First consider $I_{1}$. By forming nonoverlapping blocks of $2 m$ consecutive $\bar{W}_{m, i}^{2}-\mathrm{E} \bar{W}_{m, i}^{2}$ 's (with the last block having possibly less than $2 m$ variables) and regrouping the block sums $V_{i}$ 's (say) according to even and odd indices and using condition $M_{r}\left(r=7+2 a_{0}\right)$, one can show that

$$
\begin{aligned}
& \frac{\mathrm{E} I_{1}^{2}}{(N-m)^{2} M^{2}}\left[\mathrm{E}\left(\sum_{i \text { even }} V_{i}\right)^{2}+\mathrm{E}\left(\sum_{i \text { odd }} V_{i}\right)^{2}\right] \\
\leq & \frac{C m^{2}}{(N-m)^{2} M^{2}} \frac{M}{2 m}\left[\sum_{1 \leq i \leq M / 2 m} \alpha([i-1] 2 m+1)^{\frac{1}{3}}\right] \\
& \quad \times \max \left\{\mathrm{E}\left|\sum_{i=1}^{k}\left(\bar{W}_{m, i}^{2}-\mathrm{E} \bar{W}_{m, i}^{2}\right)\right|^{3}: 1 \leq k \leq 2 m\right\}^{\frac{2}{3}} \\
\leq & C \frac{m}{N^{2} M} m^{2}\left(\left.\mathrm{E}\left(\bar{W}_{m, 1}^{2}-\mathrm{E} \bar{W}_{m, 1}^{2}\right)\right|^{3}\right)^{\frac{2}{3}} \\
\leq & C \frac{m^{3}}{n^{3}}\left(\mathrm{E} \bar{W}_{m, 1}^{6}\right)^{\frac{2}{3}} \\
\leq & C \frac{m^{3}}{n^{3}}\left[m^{-3} \ell^{3}\left(\mathrm{E}\left\|U_{1,1}\right\|^{14}\right)^{\frac{6}{7}}\right]^{\frac{2}{3}}=O\left(m \ell^{2} / n^{3}\right),
\end{aligned}
$$

using $\mathrm{E}\left\|U_{1,1}\right\|^{14}=O(1)$ by Lemma 1 (ii) and $\sum_{j=1}^{\infty} j^{5} \alpha([j-1] 2 \ell+1)^{1 / 7}=O(1)$ by condition $M_{r}$. In the above, we have again used a regrouping of nonoverlapping blocks of $2 \ell$ consecutive $Y_{i}=\nabla^{\prime} X_{i}$ 's and splitting the sum into odd and even parts to obtain the bound on $\mathrm{E} \bar{W}_{m, 1}^{6}$.

Next consider $I_{3}$ and $I_{6}$. Note that by (B.22) and by arguments from the proof of Theorem 5, it follows that

$$
I_{3}=O\left([m / N-m] \cdot\left[n^{-1} \ell\right]\right)=O\left([m \ell] / n^{2}\right) .
$$

Also, using (B.22), one may bound $I_{6}=\left|\operatorname{Var}\left(\hat{\varphi}_{n}\right)-\frac{m}{N-m} \mathrm{E} \bar{W}_{m, 1}^{2}\right|$ by

$$
\begin{aligned}
& \left|\operatorname{Var}\left(N^{-1} \sum_{i=1}^{N} W_{i}\right)-\frac{m}{N-m} \mathrm{E} \bar{W}_{m, 1}^{2}\right|+2\left|\operatorname{Cov}\left(N^{-1} \sum_{i=1}^{N} W_{i}, \hat{R}_{1 n}^{(0)}\right)\right|+\mathrm{E}\left(\hat{R}_{1 n}^{(0)}\right)^{2} \\
& \equiv I_{61}+I_{62}+I_{63}, \quad \text { (say). }
\end{aligned}
$$


Note that using the stationarity of $X_{i}$ 's and condition $M_{r}$, we have

$$
\begin{aligned}
I_{61} \leq 2 \frac{1}{N} \sum_{k=m}^{N-1}\left|\operatorname{Cov}\left(W_{1}, W_{k+1}\right)\right|+2 \frac{1}{N} \sum_{k=1}^{m-1}\left(\frac{1}{m}+\frac{1}{N}\right)\left|\operatorname{Cov}\left(W_{1}, W_{k+1}\right)\right| \\
+C \frac{m}{N^{2}}\left[\operatorname{Var}\left(W_{1}\right)+2 \sum_{k=1}^{m-1}\left|\operatorname{Cov}\left(W_{1}, W_{k+1}\right)\right|\right] \\
\leq C \frac{1}{N} A(m-\ell)\left(\mathrm{E}\left|W_{1}\right|^{3}\right)^{\frac{2}{3}}+\frac{C}{N m}\left[\sum_{k=1}^{\ell} k \mathrm{E} W_{1}^{2}+B(1)\left(\mathrm{E}\left|W_{1}\right|^{3}\right)^{\frac{2}{3}}\right] \\
\quad+\frac{C m}{N(N-m)}\left[\sum_{k=1}^{\ell} \mathrm{E} W_{1}^{2}+A(0)\left(\mathrm{E}\left|W_{1}\right|^{3}\right)^{\frac{2}{3}}\right] \\
\leq C \frac{\ell^{2}}{n m}+C n^{-1} A(m-\ell)+C \frac{m \ell}{n^{2}}
\end{aligned}
$$

where $A(k)=\sum_{j=k}^{\infty} \alpha(j)^{1 / 3}$ and $B(k)=\sum_{j=k}^{\infty} j \alpha(j)^{1 / 3}<\infty, k \geq 0$. Also, by Lemma 1 and arguments leading to (5.4) in Lahiri (2002), $I_{63}=\mathrm{E}\left(\hat{R}_{1 n}^{(0)}\right)^{2}=O\left(n^{-1}\right)$ holds. Hence, by Cauchy-Schwarz inequality, it follows that

$$
I_{6}=O\left(\ell^{2} /[m n]\right)
$$

Next we sketch the basic steps in deriving the upper bounds on the smaller order terms. Note that in view of (B.22), we bound $I_{2}=m[(N-m) M]^{-1} \sum_{i=1}^{M} \mid N \hat{R}_{1 n}^{(0)}-(N-$ $m)\left.\hat{R}_{1 n}^{(i)}\right|^{2} / m^{2}$ by

$$
\begin{aligned}
& \frac{C}{m n} \frac{\ell^{2}}{M} \sum_{i=1}^{M}\left|N\left(\nabla^{\prime} \hat{\mu}_{n, 0}\right)^{2}-(N-m)\left(\nabla^{\prime} \hat{\mu}_{n, i}\right)^{2}\right|^{2}+ \\
& \frac{C n^{2}}{m n M} \sum_{i=1}^{M}\left|\sum_{\substack{\|\alpha\|_{1}=1 \\
\|\beta\|_{1}=2}} \frac{c_{\alpha} c_{\beta}}{p^{2}}\left[N \mathrm{E}_{*}\left(U_{1}^{* 0}-\hat{\mu}_{n, 0}\right)^{\alpha+\beta}-(N-m) \mathrm{E}_{*}\left(U_{1}^{* i}-\hat{\mu}_{n, i}\right)^{\alpha+\beta}\right]\right|^{2} \\
& +\frac{C n^{4}}{m n}\left[\left(\mathrm{E}_{*}\left\|\bar{X}_{n, 0}^{*}\right\|^{4}\right)^{2}+\left(\mathrm{E}_{*}\left\|\bar{X}_{n, 0}^{*}\right\|^{6+2 a_{0}}\right)^{2}\right] \\
& +\frac{C n^{4}}{m n}\left[\frac{1}{M} \sum_{i=1}^{M}\left\{\left(\mathrm{E}_{*}\left\|\bar{X}_{n, i}^{*}\right\|^{4}\right)^{2}+\left(\mathrm{E}_{*}\left\|\bar{X}_{n, i}^{*}\right\|^{6+2 a_{0}}\right)^{2}\right\}\right] .
\end{aligned}
$$

where $U^{* i}=U_{1, i}^{*} / \sqrt{\ell}, i=0, \ldots, M$. 
For any $\alpha, \beta, \gamma \in\left(\mathbb{Z}_{+}\right)^{d},|\alpha|=1=|\beta|,|\gamma|=2$, with $T_{i}(\alpha)=\sum_{j=i}^{i+m-1} X_{j}^{\alpha}$,

$$
\begin{aligned}
& \frac{\ell^{2}}{M} \sum_{i=1}^{M}\left[N \hat{\mu}_{n, 0}^{\alpha+\beta}-(N-m) \hat{\mu}_{n, i}^{\alpha+\beta}\right]^{2} \\
\leq & C \frac{\ell^{2}}{M} \sum_{i=1}^{M}\left[\left\{N \hat{\mu}_{n, 0}^{\alpha}-(N-m) \hat{\mu}_{n, i}^{\alpha}\right\}^{2}\left(\hat{\mu}_{n, 0}^{\beta}\right)^{2}\right] \\
& +(N-m)^{2} C \frac{\ell^{2}}{M} \sum_{i=1}^{M}\left[\left(\hat{\mu}_{n, i}^{\alpha}\right)^{2}\left[N \hat{\mu}_{n, 0}^{\beta}-(N-m) \hat{\mu}_{n, i}^{\beta}\right]^{2}\right] \\
= & C \frac{\ell^{2}}{M} \sum_{i=1}^{M} T_{i}(\alpha)^{2}\left(\hat{\mu}_{n, 0}^{\beta}\right)^{2}+C \frac{\ell^{2}}{M} \sum_{i=1}^{M}(N-m)^{2}\left(\hat{\mu}_{n, i}^{\alpha}\right)^{2}\left[\frac{T_{i}(\beta)^{2}}{N^{2}}+\frac{m^{2}}{N^{2}}\left(\hat{\mu}_{n, i}^{\beta}\right)^{2}\right] \\
= & O_{p}\left(\ell^{2} m n^{-1}\right) ; \text { and similarly, } \\
& M^{-1} \sum_{i=1}^{M}\left[N\left(\mathrm{E}_{*}\left[U_{1}^{* 0}\right]^{\gamma}\right) \hat{\mu}_{n, 0}^{\alpha}-(N-m)\left(\mathrm{E}_{*}\left[U_{1}^{* i}\right]^{\gamma}\right) \hat{\mu}_{n, i}^{\alpha}\right]^{2} \\
\leq & \frac{1}{M} \sum_{i=1}^{M} T_{i}(\alpha)^{2}\left[\mathrm{E}_{*}\left(U_{1}^{* 0}\right)^{\gamma}\right]^{2}+\left[\frac{1}{M} \sum_{i=1}^{M}\left(\hat{\mu}_{n, i}^{\alpha}\right)^{4}\right]^{1 / 2} \times \\
& \left\{\frac{1}{M} \sum_{i=1}^{M}\left(\sum_{j=i}^{i+m-1}\left(U_{j}^{\gamma}-\mathrm{E} U_{1}^{\gamma}\right)\right)^{4}+\frac{m^{2}}{N^{2} M} \sum_{i=1}^{M}\left(\sum_{j \in I_{i}}\left(U_{j}^{\gamma}-\mathrm{E} U_{1}^{\gamma}\right)\right)^{4}\right\}^{1 / 2} \\
= & O_{p}\left(m \ell^{-2}\right)+O_{P}\left(n^{-1}\left\{\ell^{-4}\left[\frac{m}{\ell} \ell^{2}\right]^{2}+\frac{m^{2}}{n^{2}} \ell^{-4} n^{2}\right\}^{1 / 2}\right)=O_{p}\left(m \ell^{-2}\right),
\end{aligned}
$$

for $U_{j}=\left(X_{j}+\cdots+X_{j+\ell-1}\right) / \ell, j \geq 1$.

Also, noting that $\mathrm{E}_{*}\left\|U_{1}^{* i}\right\|^{r} \leq[N /(N-m+1)] \mathrm{E}_{*}\left\|U_{1}^{* 0}\right\|^{r}$ for all $r>0$ and for all $i=1, \ldots, M$, we get

$$
\begin{aligned}
\frac{1}{M} \sum_{i=1}^{M}\left[\mathrm{E}_{*}\left\|\bar{X}_{n, i}\right\|^{6+2 a_{0}}\right]^{2} & \leq C \frac{1}{M} \sum_{i=1}^{M}\left[\frac{1}{p^{3+a_{0}}} \mathrm{E}_{*}\left\|U_{1}^{* i}\right\|^{6+2 a_{0}}\right]^{2} \\
& \leq C \frac{1}{p^{6+2 a_{0}}}\left[\frac{1}{N} \sum_{j=1}^{N}\left\|U_{j}\right\|^{6+2 a_{0}}\right]^{2}=O_{p}\left(n^{-6-2 a_{0}}\right)
\end{aligned}
$$

by condition $M_{r}$, Lemma 1 (ii) and $p=n / \ell$.

Now, using the bounds above and (B.25), one can show that

$$
I_{2}=O_{p}\left(\frac{\ell^{2}}{n^{2}}+\frac{1}{m n}+\frac{m}{n^{2}}\right) .
$$

By similar arguments, $I_{4}$ is also negligible. And for $I_{5}$, it is easy to check that there exist

imsart-bj ver. 2011/12/06 file: BEJ511.supplementary.material.tex date: February 8, 2013 
weights $\left\{w_{\text {in }}: 1 \leq i \leq M+\ell-1\right\} \in[0,1]$ such that

$$
\begin{aligned}
I_{5} & \leq C n^{-1} m \cdot\left|\hat{\varphi}_{n}-\mathrm{E} W_{1}\right| \cdot\left|M^{-1} \sum_{i=1}^{M+\ell-1} w_{i n}\left(W_{i}-\mathrm{E} W_{i}\right)\right| \\
& =C n^{-1} m \cdot O_{p}\left(p^{-1 / 2}\right) \cdot O_{p}\left(p^{-1 / 2}\right)=O_{p}\left(\frac{m}{n} \cdot \frac{\ell}{n}\right),
\end{aligned}
$$

where the bound on the last term in the first line follows by computing its second moment (using even and odd numbered blocks of $2 \ell$-many $w_{i n}\left(W_{i}-\mathrm{E} W_{i}\right)$ 's) as in the derivation of (B.23).

Now combining the bounds on $I_{1}-I_{6}$, with $p=n / \ell$,

$$
\left.\left|\widehat{\mathrm{VAR}}-\operatorname{Var}\left(\hat{\varphi}_{n}\right)\right|=O_{p}\left(p^{-1}[m / n]^{1 / 2}+\ell / m\right]\right)
$$

Next using the arguments in the proof of Theorem 5(ii), one can show that

$$
\begin{aligned}
\operatorname{Var}\left(\hat{\sigma}_{n}^{2}(\ell)\right) & =\operatorname{Var}\left(p \ell \operatorname{Var}\left(L_{n}^{*}\right)\right)+O\left(n^{-2}\right) \\
& =\operatorname{Var}\left(\ell N^{-1} \sum_{i=1}^{N}\left[W_{i} / \sqrt{\ell}\right]^{2}\right)+O\left(n^{-1} \ell^{-1}\right) \\
& =\frac{V_{0}}{p}+O\left(n^{-1} \ell^{-1}\right)
\end{aligned}
$$

Hence, by (B.27) and (B.28), it follows that

$$
\begin{aligned}
\left|\hat{V}_{0}-V_{0}\right| & =\left|n \ell^{-1} \widehat{\mathrm{VAR}}-V_{0}\right| \\
& \leq\left|n \ell^{-1}\left(\widehat{\mathrm{VAR}}-\operatorname{Var}\left(\hat{\sigma}_{n}^{2}(\ell)\right)\right)\right|+\left|n \ell^{-1} \operatorname{Var}\left(\hat{\sigma}_{n}^{2}(\ell)\right)-V_{0}\right| \\
& =O_{p}\left([m / n]^{1 / 2}+[\ell / m]+\ell^{-2}\right)
\end{aligned}
$$

This establishes the error contribution of $\left|\hat{V}_{0}-V_{0}\right|$ in Theorem 3 with $\ell_{1}=\ell$. 